\title{
The biological activity of FasL \\ in human and mouse lungs is determined by the structure of its stalk region
}

Raquel Herrero,1,2 Osamu Kajikawa,1,2 Gustavo Matute-Bello, 2,3 Yi Wang, 4,5 Naoki Hagimoto, 1,2 Steve Mongovin, ${ }^{1,2}$ Venus Wong, ${ }^{1,2}$ David R. Park, ${ }^{2}$ Nathan Brot, ${ }^{6}$ Jay W. Heinecke,7 Henry Rosen, ${ }^{8}$ Richard B. Goodman,, ${ }^{1,2}$ Xiaoyun Fu, ${ }^{4,5}$ and Thomas R. Martin ${ }^{1,2}$

\author{
${ }^{1}$ Medical Research Service of the VA Puget Sound Health Center, ${ }^{2}$ Division of Pulmonary and Critical Care Medicine, \\ ${ }^{3}$ Center for Lung Biology, Division of Pulmonary and Critical Care Medicine, and ${ }^{2}$ Division of Hematology, \\ Department of Medicine, University of Washington, Seattle, Washington, USA. 5Puget Sound Blood Center, Seattle, Washington, USA. \\ ${ }^{6}$ Department of Microbiology and Immunology, Weill Medical College of Cornell University, New York, New York, USA. \\ ${ }^{7}$ Division of Metabolism, Endocrinology and Nutrition, and ${ }^{8}$ Division of Allergy and Infectious Diseases, \\ Department of Medicine, University of Washington, Seattle, Washington, USA.
}

\begin{abstract}
Acute lung injury (ALI) is a life-threatening condition in critically ill patients. Injury to the alveolar epithelium is a critical event in ALI, and accumulating evidence suggests that it is linked to proapoptotic Fas/FasL signals. Active soluble FasL (sFasL) is detectable in the bronchoalveolar lavage (BAL) fluid of patients with ALI, but the mechanisms controlling its bioactivity are unclear. We therefore investigated how the structure of sFasL influences cellular activation in human and mouse lungs and the role of oxidants and proteases in modifying sFasL activity. The sFasL in BAL fluid from patients with ALI was bioactive and present in high molecular weight multimers and aggregates. Oxidants generated from neutrophil myeloperoxidase in BAL fluid promoted aggregation of sFasL in vitro and in vivo. Oxidation increased the biological activity of sFasL at low concentrations but degraded $\mathrm{sFasL}$ at high concentrations. The amino-terminal extracellular stalk region of human $\mathrm{SFasL}$ was required to induce lung injury in mice, and proteolytic cleavage of the stalk region by MMP-7 reduced the bioactivity of sFasL in human cells in vitro. The sFasL recovered from the lungs of patients with ALI contained both oxidized methionine residues and the stalk region. These data provide what we believe to be new insights into the structural determinants of sFasL bioactivity in the lungs of patients with ALI.
\end{abstract}

\section{Introduction}

Acute lung injury (ALI), including its more severe form, acute respiratory distress syndrome (ARDS), is common in critically ill patients and carries an overall mortality of $40 \%$ or higher in unselected patients (1). The pathological hallmark of ALI and ARDS is diffuse alveolar damage with protein-rich edema, in which alveolar epithelial injury is a critical event (2-4). Epithelial injury in ALI occurs by necrosis and apoptosis $(2,5)$, but the mechanisms that mediate these events in the alveolar epithelium are not completely understood. Apoptosis is a regulated form of cell death that is initiated by membrane death receptors and/or direct mitochondrial injury $(6,7)$. Accumulating evidence in humans and experimental models links the proapoptotic Fas/FasL system with lung epithelial injury and fibrosis $(6,8)$.

Fas is a $45-\mathrm{kDa}$ type I cell surface receptor that belongs to the TNF receptor family. Binding of FasL to membrane Fas induces apoptosis in susceptible cells and activates inflammatory responses. Several lines of evidence show that Fas activation initiates proapoptotic and proinflammatory pathways in lung epithelial cells (9-14). FasL exists in both membrane and soluble forms. The membrane FasL (mFasL) is a 37-kDa type II glycoprotein that acts locally through cell-to-cell contact (15-17). The extracellular domain of the human mFasL is composed of the stalk region (103-136 aa) at the $\mathrm{NH}_{2}$-terminus close to the cell membrane and a TNF

Conflict of interest: The authors have declared that no conflict of interest exists. Citation for this article: J Clin Invest. 2011;121(3):1174-1190. doi:10.1172/JCI43004 homology domain (137-281 aa) at the COOH-terminus (18). The extracellular domain of $\mathrm{mFasL}$ can be processed by the proteolytic action of MMPs, such as MMP-7 and MMP-3, into a 25- to 29-kDa soluble form of FasL (sFasL) $(19,20)$. Preformed sFasL also can be released by activated monocytes (21).

The biological activity of sFasL varies among studies, which could be explained by differences in molecular conformation. For example, some evidence suggests that the bioactivity of sFasL depends on the formation of aggregates or multimers of sFasL molecules, as multimers of sFasL promote more efficient aggregation and activation of membrane Fas receptor in vitro (22-25). In addition, variants of sFasL with different $\mathrm{N}$-terminal amino acid sequences have been used in different studies. More information is needed about the structural factors that regulate the biological activity of SFasL in vivo.

In prior studies, we found that while sFasL is detectable before and after the onset of ARDS, only sFasL present in the bronchoalveolar lavage (BAL) fluid from patients with established ARDS induces apoptosis in human distal lung epithelial cells in vitro, suggesting that specific mechanisms control the biological activity of sFasL in the lungs in vivo (26). We hypothesized that factors in the alveolar microenvironment of patients with ARDS modulate the biological activity of sFasL by promoting changes in its structure or physical state. For example, the lungs of patients with ARDS contain a highly oxidative environment, due in large part to the accumulation of neutrophils and the release of reactive oxygen and nitrogen species (27-31). Oxidation, chlorination, and nitra- 
A
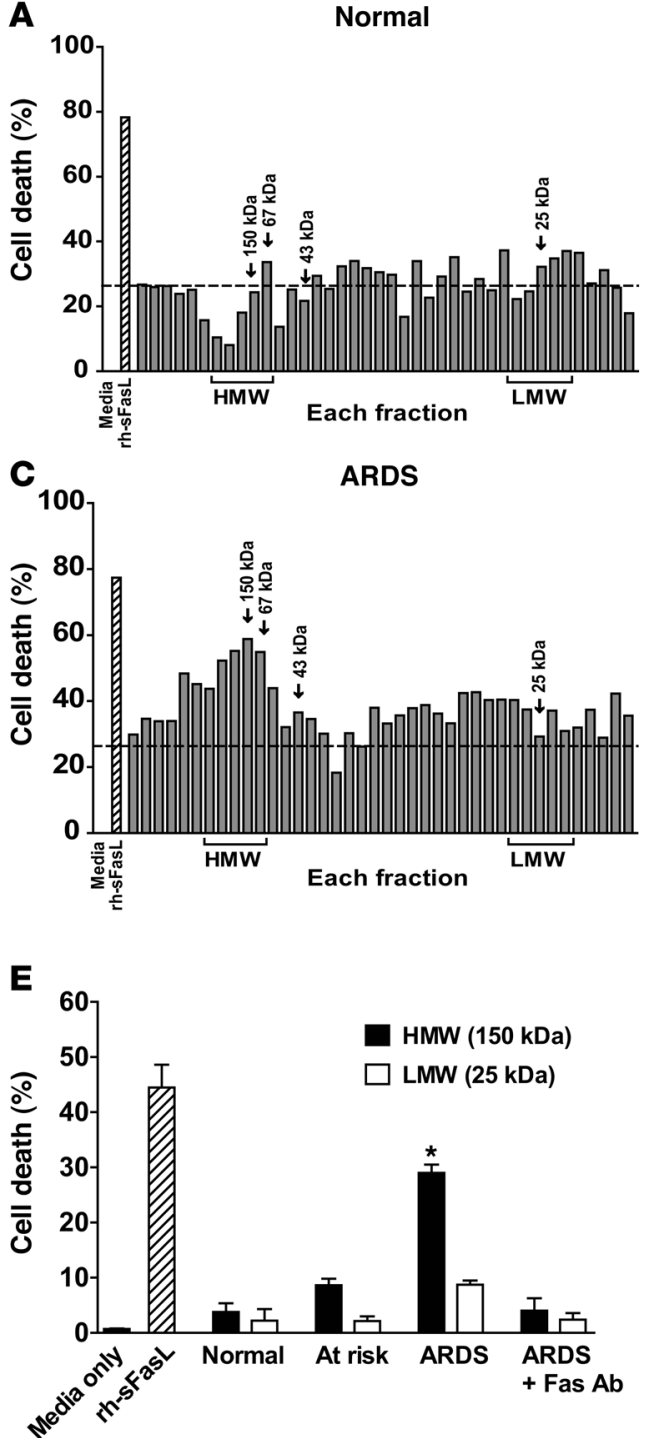

G

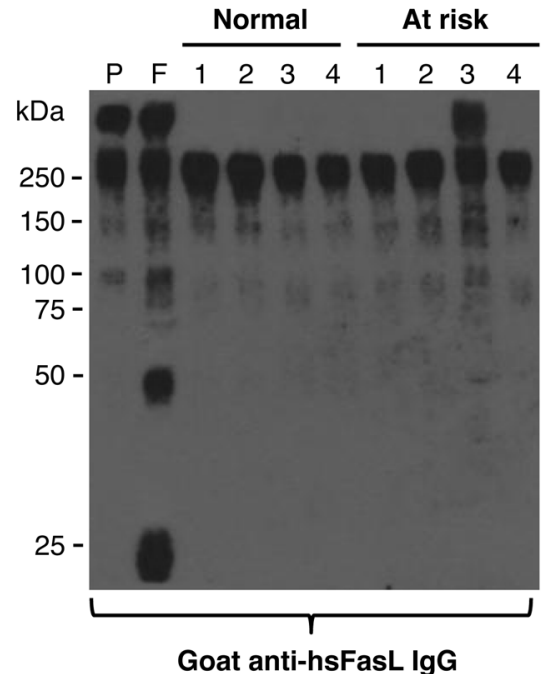

B

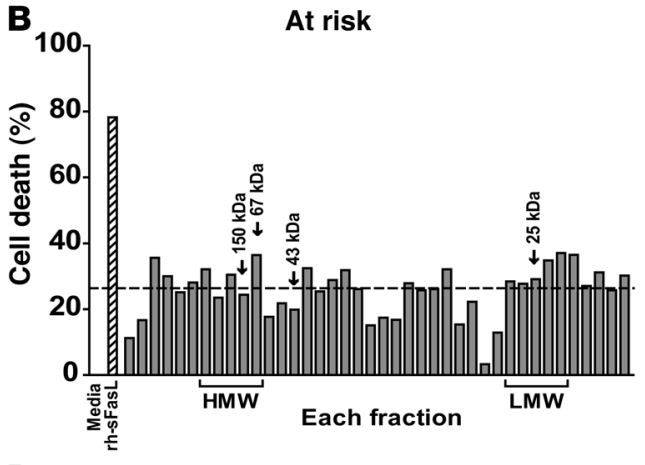

D

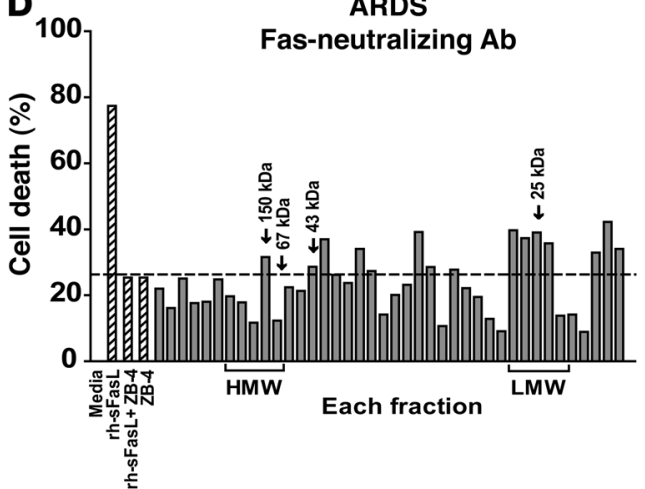

F
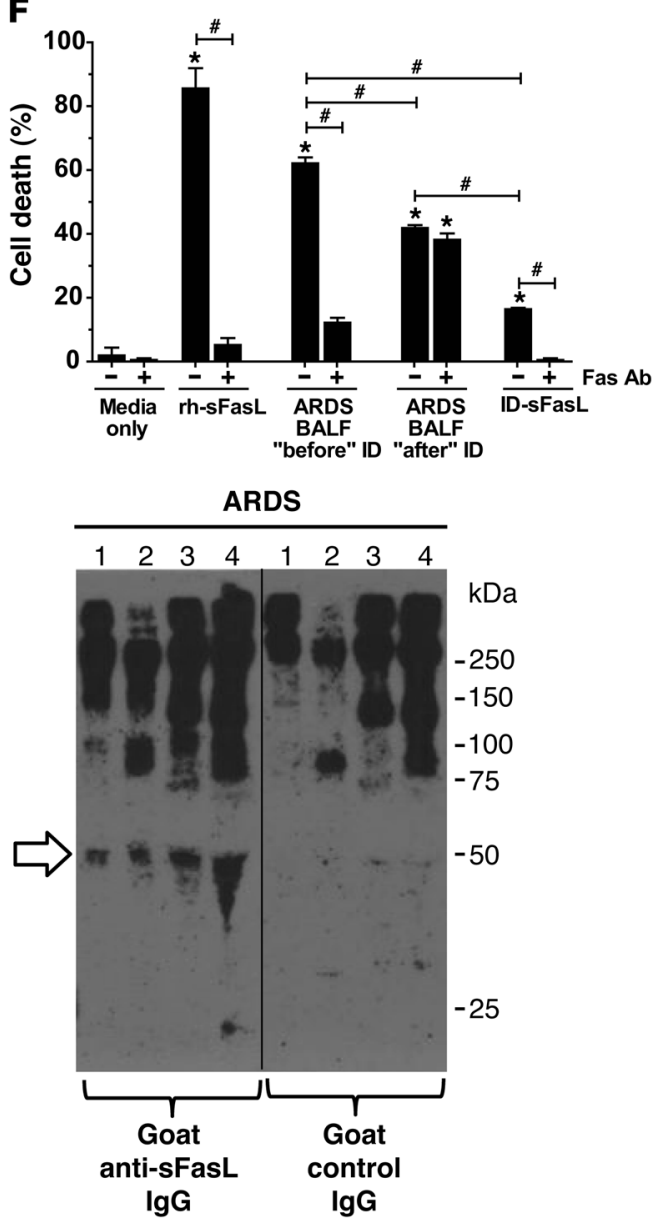

Figure 1

Aggregation and biological activity of SFasL in BAL fluid. Pooled BAL fluid from (A) 2 normal volunteers, (B) 3 at-risk patients, and (C) 4 patients with ARDS was concentrated and separated by size-exclusion HPLC, and cytotoxicity of each fraction was tested on Jurkat cells (high molecular weight [HMW], $\geq 67 \mathrm{kDa}$; low molecular weight [LMW], $22 \mathrm{kDa}$ to $28 \mathrm{kDa}$ ). (D) Effect of a Fas-neutralizing Ab on cytotoxicity of each fraction. (A-D) Dotted lines represent the mean percentage of cell death induced by normal BAL fluid. (E) Cytotoxicity of ARDS BAL fluid fractions containing high molecular weight $(150 \mathrm{kDa})$ and low molecularweight ( $25 \mathrm{kDa})$ proteins was measured for human SAECs, with or without a Fas-neutralizing Ab. ${ }^{*} P<0.01$ vs. all other samples. (F) sFasL was immunodepleted from pooled ARDS BAL fluid, and cytotoxicity was measured in Jurkat cells for original BAL fluid ("before" ID), immunodepleted BAL fluid ("after" ID), and purified SFasL in the column eluate (ID-sFasL), with or without Fas-neutralizing Ab. BALF, BAL fluid. ${ }^{*} P<0.01$ vs. media only; ${ }^{\prime} P<0.01$. (E and $\mathbf{F}$ ) Results are mean \pm SD of 3 separate experiments. (G) Western blot of 10-fold concentrated BAL fluids (nonreducing conditions). Lanes correspond to individual patients. Plasma samples from a normal volunteer without (lane P) or with rh-sFasL (hsFasL) (lane F) were included to control for cross-reactivity with plasma proteins. BAL fluid from patients with ARDS, but not that from at-risk patients or normal volunteers, contained dimers of endogenous sFasL (white arrow). High molecular weight bands $(\geq 75 \mathrm{kDa})$ are nonspecific. rh-sFasL was used as positive control for cytotoxicity. The hairline separates noncontiguous lanes of the same gel. 
A

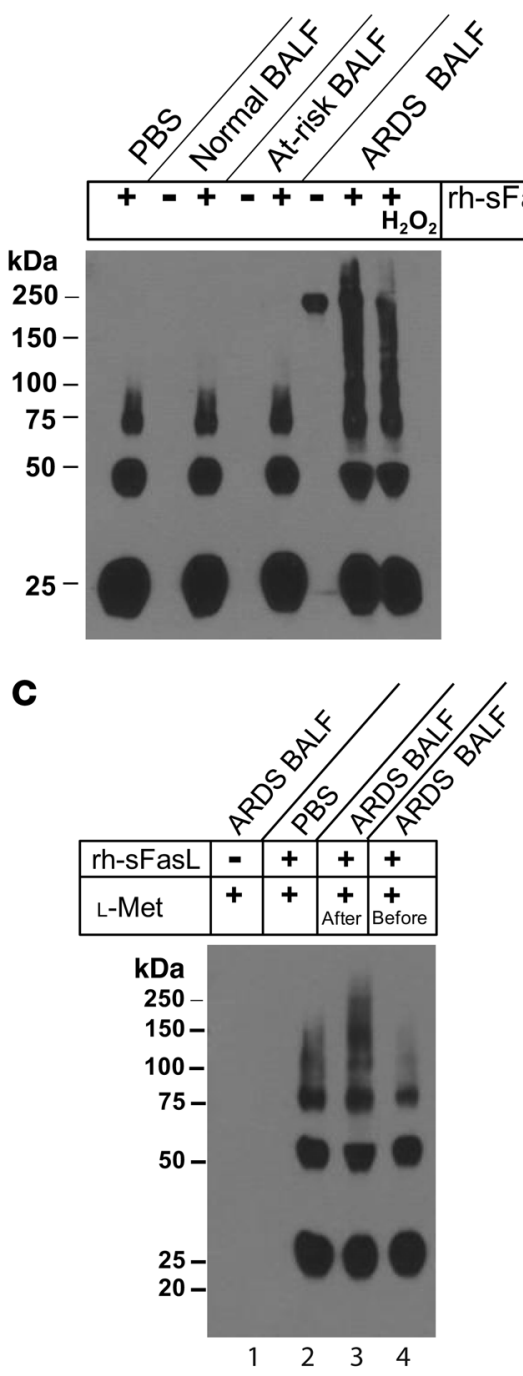

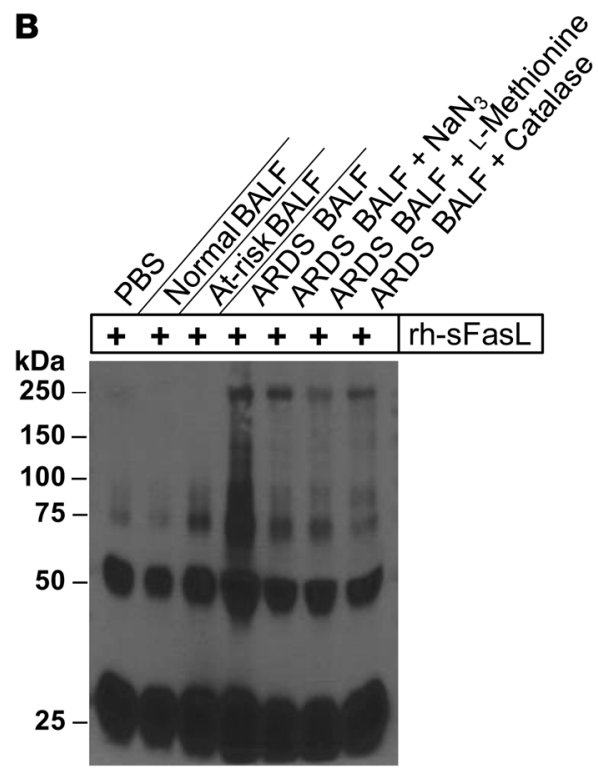

D

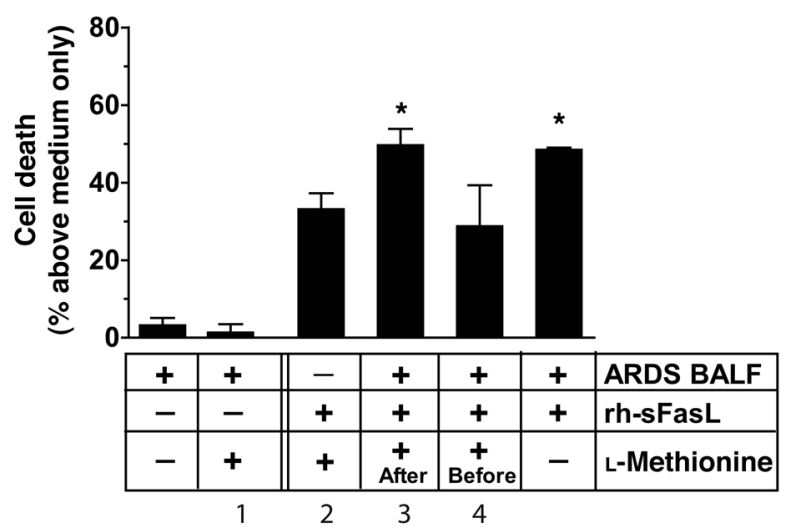

Figure 2

Effect of ARDS BAL fluid on multimerization and bioactivity of sFasL. (A) Pooled BAL fluids from 3 normal volunteers, 3 at-risk patients, and 3 patients with ARDS were diluted in PBS, incubated alone or with rh-sFasL, and then analyzed by Western blot. Larger aggregates of rh-sFasL, ranging from $100 \mathrm{kDa}$ to $250 \mathrm{kDa}$, were detected only when rh-sFasL was incubated in ARDS BAL fluid. A single 250-kDa band is present in the unspiked ARDS BAL fluid and represents either cross-reactivity or large aggregates of endogenous sFasL. (B) Effect of inhibitors (NaN ${ }_{3}$ [MPO inhibitor, $1 \mathrm{mM}$ ], L-methionine [ $\mathrm{HOCl}$ inhibitor, $500 \mu \mathrm{M}$ ], and catalase $\left[\mathrm{H}_{2} \mathrm{O}_{2}\right.$ inhibitor, $500 \mathrm{U} / \mathrm{ml}$ ]) on multimerization of rh-sFasL in ARDS BAL fluid. (C and D) Two samples of pooled ARDS BAL fluids were incubated with exogenous rh-sFasL for 90 minutes. In 1 of the ARDS BAL samples, the antioxidant L-methionine (L-met) was added after incubation with exogenous rh-sFasL (L-methionine, after). The other sample was preincubated with L-methionine, followed by incubation with exogenous rh-sFasL (L-methionine, before). Incubation of rh-sFasL in ARDS BAL fluid increased (C) multimerization and (D) cytotoxicity for Jurkat cells, which was prevented by preincubation of ARDS BAL fluid with L-methionine. (D) Low cytotoxicity of the ARDS BAL fluids is explained by dilution of the BAL fluid. Results represent mean \pm SD of 3 separate experiments performed in duplicates. ${ }^{\star} P<0.05$ vs. all other samples. All Western blots were run in nonreducing conditions. Lane numbers in $\mathbf{D}$ correspond to lane numbers in $\mathbf{C}$.

tion of amino acid residues, such as cysteine, methionine, tyrosine, and tryptophan, modify protein-protein interactions and protein activity (32-34). Therefore, we tested whether endogenous oxidants in the lungs of patients with ARDS modify the bioactivity and the degree of aggregation of sFasL. Because injured lungs also contain a proteolytic environment, we hypothesized that the bioactivity of the sFasL in the lungs is modified by proteolytic cleavage of the N-terminal domain or other sites of sFasL molecule. Finally, we analyzed the structure and degree of oxidation of endogenous sFasL derived from the lungs of humans with ALI.

Herein we show that aggregates of sFasL are detectable in the BAL fluid from patients after the onset of ARDS, but not in that from at-risk patients, and that multimerization of $\mathrm{sFasL}$ is associated with methionine oxidation via the MPO- $\mathrm{H}_{2} \mathrm{O}_{2}$-chloride 
A

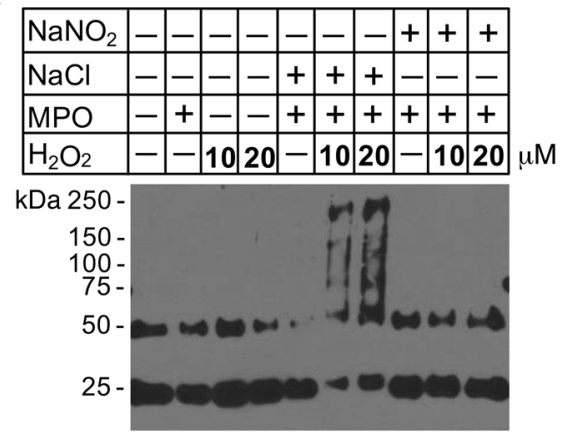

C

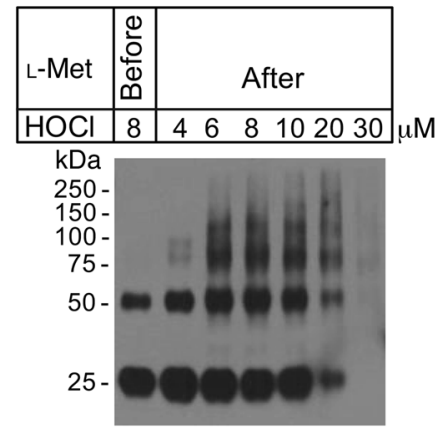

D

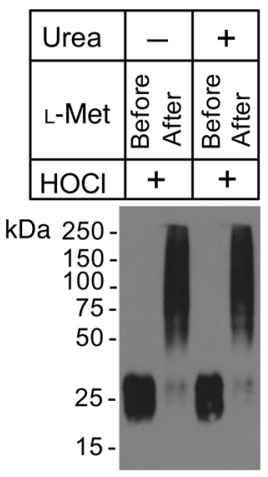

B

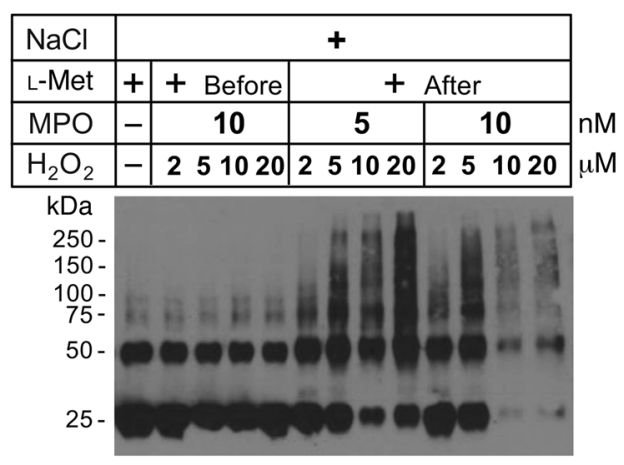

E

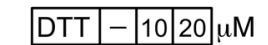

F

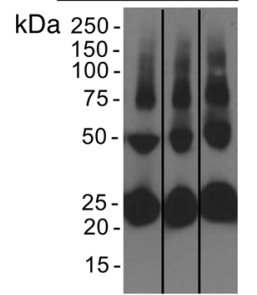

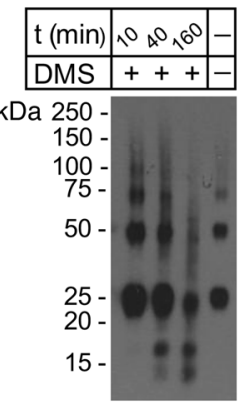

\section{Figure 3}

Effect of oxidants on multimerization of sFasL. (A) Western blot analysis of rh-sFasL incubated in PBS-DTPA alone or in the presence of $\mathrm{H}_{2} \mathrm{O}_{2}$ $(10 \mu \mathrm{M}$ or $20 \mu \mathrm{M})$ with or without MPO $(50 \mathrm{nM})$ and either $\mathrm{NaNO}_{2}(100 \mu \mathrm{M})$ or $\mathrm{NaCl}(100 \mathrm{mM})$. Reactions were terminated at 60 minutes with L-methionine $(2.5 \mathrm{nM})$. Multimers of rh-sFasL (75-kDa and larger molecular weight bands) were detected when sFasL was exposed to MPO$\mathrm{H}_{2} \mathrm{O}_{2}$-chloride. (B and C) Dose-dependent effects of MPO $(n M)$ and $\mathrm{H}_{2} \mathrm{O}_{2}(\mu \mathrm{M})$ or $\mathrm{HOCl}(\mu \mathrm{M})$ on multimerization of rh-sFasL, respectively. Degradation of rh-sFasL occurred at the highest concentrations of MPO and $\mathrm{H}_{2} \mathrm{O}_{2}$ or $\mathrm{HOCl}$. Western blot analyses in $\mathbf{A}-\mathbf{C}$ were performed using SDS-PAGE with 2-ME and heat. (D) Lack of effect of urea (8 M) on oxidized multimers of rh-sFasL. Samples were resolved in SDS-PAGE with 2-ME (no heat). (E) Lack of effect of DTT (10 $\mu \mathrm{M}$ or $20 \mu \mathrm{M})$ on HOCl-oxidized multimers of rh-sFasL. Western blot was performed in SDS-PAGE with no 2-ME or heat. $(\mathbf{F})$ Effect of DMS and $\mathrm{HCl}$ on multimerization of $\mathrm{HOCl}$-oxidized multimers of rh-sFasL. Samples were filtered by centrifugation to eliminate DMS and $\mathrm{HCl}$ and neutralize $\mathrm{pH}$ and then subjected to SDS-PAGE in nonreducing conditions (no 2-ME, no heat). In $\mathbf{D}-\mathbf{F}$, the lanes were taken from the same gels. The vertical hairlines in $\mathbf{E}$ separate noncontiguous lanes in the same gel.

system in the ARDS BAL fluid but not with nitration of sFasL. In addition, we report that the presence of the $\mathrm{N}$-terminal stalk region of sFasL has a previously unrecognized role in the biological activity of sFasL in vivo and that endogenous sFasL in the lungs of patients with ALI contains the stalk region and oxidized methionine residues. The data suggest that the bioactivity of sFasL in the lungs of humans is determined by the $\mathrm{N}$-terminal site at which $\mathrm{mFasL}$ is cleaved and the extent of methionine oxidation.

\section{Results}

ARDS BAL fluid contains biologically active sFasL in high molecular weight fractions. We investigated whether endogenous $\mathrm{SFasL}$ aggregates in the BAL fluid from patients with ARDS and, if so, whether aggregation influences the biological activity of sFasL. The BAL fluid from 2 normal volunteers, 3 patients at risk for ARDS, and 4 patients with ARDS was fractionated using size-exclusion chromatography, and then the cytotoxicity of each molecular weight fraction was tested using human Jurkat cells, which are sensitive to sFasL. The percentage of cell death in Jurkat cells exposed to normal and at-risk BAL fluid fractions ranged from $8.1 \% \pm 17.7 \%$ to $37.3 \% \pm 9.8 \%$ and from $3.3 \% \pm 4.1 \%$ to $37.1 \% \pm 15.2 \%$, respectively (Figure 1, A and B), whereas cell death increased up to
$58.8 \% \pm 4.5 \%$ when Jurkat cells were incubated with the high molecular weight fractions ( $67 \mathrm{kDa}$ to $>150 \mathrm{kDa}$ ) from ARDS BAL fluid (Figure 1C). Blocking Fas on Jurkat cells using the ZB4 anti-Fas antibody reduced the cytotoxicity of the higher molecular weight fractions of the ARDS BAL fluid (Figure 1D), supporting the conclusion that the cytotoxicity was due predominantly to sFasL in the BAL fluid fractions. Compared with the high molecular weight fractions, the low molecular weight fractions ( $22 \mathrm{kDa}$ to $28 \mathrm{kDa}$ ) of ARDS BAL fluid, which should contain monomeric forms of sFasL $(25 \mathrm{kDa})$, induced significantly less cell death $(P<0.05)$.

To confirm the relevance of these data in human lung epithelial cells, primary human small airway epithelial cells (SAECs) were incubated for 18 hours in medium only, medium supplemented with $500 \mathrm{ng} / \mathrm{ml}$ recombinant human sFasL (rh-sFasL) (as positive control), and medium supplemented with either the high molecular weight fraction $(150 \mathrm{kDa})$ or the low molecular weight fraction $(25 \mathrm{kDa})$ from the pooled BAL fluids (Figure 1E). Incubation with pooled BAL fluid from normal or at-risk patients resulted in minimal cell death. In contrast, incubation with the $150-\mathrm{kDa}$ ARDS fluid fraction resulted in $29.0 \% \pm 2.6 \%$ cell death $(P<0.05$, compared with all other conditions), and this was blocked by the 

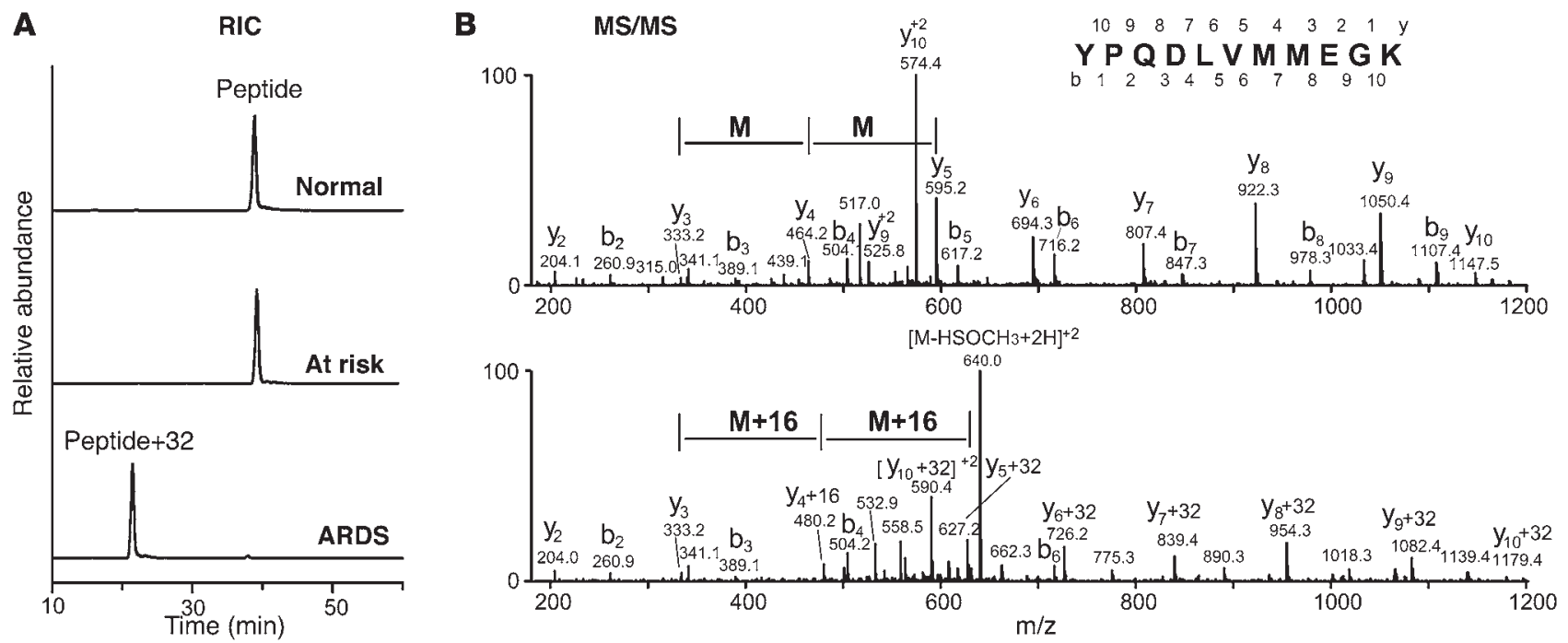

Figure 4

Oxidation of methionine residues in sFasL by ARDS BAL fluid. (A) Reconstructed ion chromatogram (RIC) of a tryptic peptide from rh-sFasL exposed to normal, at-risk, or ARDS BAL fluid. The mass-to-charge ratios of the peptide from sFasL exposed to normal and at-risk BAL fluid were 656.1. The mass-to-charge ratio of the peptide from sFasL exposed to ARDS BAL fluid was 672.1 (original peptide $+32 \mathrm{Da}$, doubly charged). The increase of $32 \mathrm{Da}$ corresponds to the addition of 2 oxygen atoms to the peptide. (B) MS/MS analysis of peptide Y218PQDLVMMEGK (top row) and oxidized peptide $\mathrm{Y}^{218} \mathrm{PQDLVM}(\mathrm{O}) \mathrm{M}(\mathrm{O}) \mathrm{EGK}$ (bottom row) in a tryptic digest of rh-sFasL exposed to normal BAL fluid (top row) or ARDS BAL fluid. Exposure to ARDS fluid resulted in oxidation of the 2 methionine residues near the receptor-ligand binding site $\left(\mathrm{Y}^{218}\right)$.

anti-Fas neutralizing antibody. The 25-kDa ARDS fluid fraction induced minimal cell death $(P<0.05$, compared with the ARDS high molecular weight fraction).

To further confirm that the cytotoxicity of ARDS BAL fluid is due to the action of sFasL, pooled BAL fluid from 4 patients with ARDS was immunodepleted of sFasL using an immunoaffinity column containing an anti-human sFasL mAb (Figure 1F). The concentrations of the endogenous sFasL in the ARDS BAL fluid, before and after the immunodepletion, were 714 and $114 \mathrm{pg} / \mathrm{ml}$, respectively, and the concentration of the purified sFasL in the column eluate was $969 \mathrm{pg} / \mathrm{ml}$. The ARDS BAL fluid induced $62.1 \% \pm 1.9 \%$ cell death. This cytotoxicity was blunted by blocking the Fas receptor on the Jurkat cells $(12.2 \% \pm 1.5 \%$; $P<0.05)$ and was also significantly reduced by immunodepletion of sFasL $(41.8 \% \pm 0.9 \% ; P<0.05)$. The cytotoxicity of the immunodepleted ARDS BAL fluid was not further reduced with the anti-Fas receptor $\mathrm{mAb}(38.2 \% \pm 1.9 \%$ cell death), suggesting the presence of other cytotoxic factors in addition to sFasL in the ARDS BAL fluid. The material eluted from the column was cytotoxic $(16.4 \% \pm 0.5 \%)$ and almost completely inhibitable by blocking Fas on Jurkat cells, consistent with sFasL. Altogether, these data show that ARDS BAL fluid induces cell death by mechanisms involving activation of the Fas/FasL pathway, although, other pathways also appear to be involved.

Human sFasL aggregates in the lungs of patients with ARDS. To further define the molecular size of sFasL in ARDS BAL fluid, we performed Western blot with nonreducing SDS-PAGE using concentrated BAL fluid from normal volunteers, patients at risk for ARDS, and patients with established ARDS. Initial experiments showed substantial cross-reactivity among several different HRP-conjugated secondary antibodies and other proteins present in the BAL fluid. To minimize cross-reactivity, the BAL fluid samples were immunodepleted of albumin and IgG. Never- theless, bands ranging from $100 \mathrm{kDa}$ to $250 \mathrm{kDa}$ were present in normal plasma and BAL fluid samples from normal volunteers, patients at risk for ARDS, and patients with ARDS, even when a control antibody was used (Figure 1G), showing that these bands are not specific for sFasL. Normal plasma spiked with recombinant sFasL showed bands at 25, 50, and $75 \mathrm{kDa}$, corresponding to monomers, dimers, and trimers of sFasL (Figure $1 \mathrm{G}$, lane F). Dimers of sFasL were detected in BAL fluid samples from all patients with ARDS but were not detected in the BAL fluid from normal volunteers or patients at risk for ARDS. Monomers of sFasL were not detected in any of the samples.

ARDS BAL fluid multimerizes rh-sFasL. To determine whether substances present in the BAL fluid influence the multimerization of sFasL, rh-sFasL was incubated in PBS or BAL fluid from normal subjects, at-risk patients, or patients with established ARDS (Figure 2). Western blot of the rh-sFasL incubated in PBS showed bands at 25 and $50 \mathrm{kDa}$ and a less intense band at $75 \mathrm{kDa}$, consistent with the predicted molecular weight of glycosylated monomers, dimers, and trimers, respectively (Figure 2A). When rh-sFasL was incubated in BAL fluid from patients with ARDS, additional bands appeared at higher molecular weights, ranging from $100 \mathrm{kDa}$ to $250 \mathrm{kDa}$, indicating the formation of SDS-resistant aggregates of sFasL.

Effect of oxidants on multimerization and bioactivity of sFasL in ARDS BAL fluid. Hypochlorous acid ( $\mathrm{HOCl})$, generated from $\mathrm{H}_{2} \mathrm{O}_{2}$ and $\mathrm{Cl}^{-}$via the catalytic action of myeloperoxidase (MPO) in activated neutrophils and monocytes, is a potent oxidant that can alter the structure and biological activity of proteins (32, $35,36)$. Because both MPO and $\mathrm{H}_{2} \mathrm{O}_{2}$ accumulate in the lung fluids of patients with $\operatorname{ARDS}(27,30)$, we investigated the effect of oxidants on the multimerization of added rh-sFasL, using the same pooled BAL fluid samples tested previously. Aggregation of rh-sFasL was observed only when rh-sFasL was incubated in 
A

Mouse Native-sFasL

-_-_-_-_ - -

PHSRSIPLEW EDTYGTALIS GVKYKKGGLV INETGLYFVY SKVYFRGQSC 200 NNQPLNHKVY MRNSKYPEDL VLMEEKRLNY CTTGQIWAHS SYLGAVFNLT 250 SADHLYVNIS QLSLINFEES KTFFGLYKL

Mouse Met-sFasL

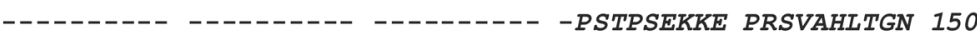

PHSRSMPLEW EDTYGTALIS GVKYKKGGLV INETGLYFVY SKVYFRGQSC 200

NNQPLNHKVY MRNSKYPEDL VMMEEKMMNY CTTGQMWAHS SYLGAVFNLT 250

SADHLYVNIS QLSLINFEES KTFFGLYKL

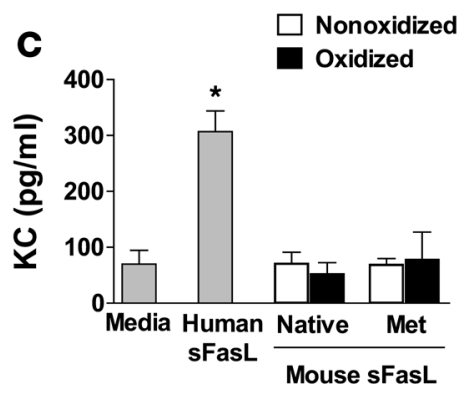

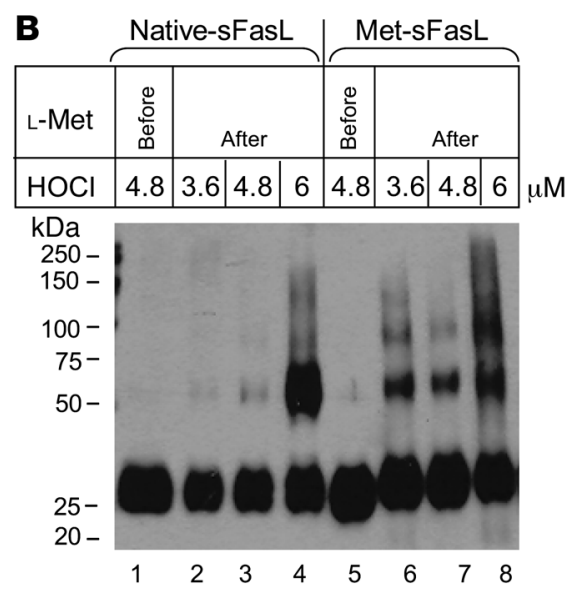

\section{Figure 5}

Methionine residues are involved in oxidative multimerization of sFasL. (A) Amino acid sequence of mouse native-sFasL and met-sFasL. Native sFasL contains 2 methionine residues (underlined), whereas met-sFasL contains 7 methionine residues (underlined) in the same locations as those in human sFasL. (B) Western blot analysis of the native form of mouse sFasL (native-sFasL) and a mutated form of mouse sFasL containing methionines at the location of methionine in human sFasL (mouse met-sFasL). Native sFasL (lanes 2-4) and met-sFasL (lanes 6-8) were incubated with increasing concentrations of $\mathrm{HOCl}(\mu \mathrm{M})$, and the reaction was stopped with L-methionine $(2.5 \mathrm{nM})$. For comparison, L-methionine was added to the reaction before incubation with mouse native sFasL (lane 1) or met-sFasL (lane 5) to prevent protein oxidation. Nonoxidized mouse native sFasL and met-sFasL showed single bands at $25 \mathrm{kDa}$, corresponding to sFasL monomers (lanes 1 and 5). When oxidized, met-sFasL (lanes 6-8) multimerized more readily than native-sFasL (lanes 2-4). (C) Biological activity of normal and oxidized forms of mouse native sFasL and met-sFasL for mouse lung epithelial cells (MLE-15). Either media alone or rh-sFasL were used as controls. Bars show the mean \pm SD of 3 separate experiments. ${ }^{*} P<0.05$ compared with all other samples. The Western blot was run in SDS-PAGE in reducing conditions (2-ME and heat).

ARDS BAL fluid (Figure 2, A-C). This was inhibited by the MPO inhibitor $\mathrm{NaN}_{3}$, the $\mathrm{H}_{2} \mathrm{O}_{2}$ scavenger catalase, and the $\mathrm{HOCl}$ scavenger L-methionine (Figure 2B).

To determine whether oxidants in the ARDS BAL fluid influence the biological activity of sFasL, exogenous rh-sFasL was incubated for 90 minutes in ARDS BAL fluid or PBS, and the oxidant scavenger L-methionine was added either before or after incubation with the rh-sFasL. As expected, the rh-sFasL incubated in ARDS BAL fluid showed a higher degree of multimerization in nonreducing conditions (Figure 2C) and also was more cytotoxic for Jurkat cells as compared with rh-sFasL incubated in PBS $(49.8 \% \pm 3.8 \%$ vs. $33.2 \% \pm 4.1 \%$ cell death, respectively; $P<0.05$ ) (Figure $2 \mathrm{D}$ ). This increase in multimerization and cytotoxicity of rh-sFasL was prevented when the antioxidant L-methionine was added to the ARDS BAL fluid prior to incubation with rh-sFasL $(27.1 \% \pm 11.2 \%$ cell death). Thus, oxidants in ARDS BAL fluid cause multimerization/ aggregation of sFasL and enhance biological activity.

To study the effects of MPO-catalyzed oxidation and nitration reactions on multimerization of human $\mathrm{sFasL}$, we exposed rh-sFasL to the MPO- $\mathrm{H}_{2} \mathrm{O}_{2}$-chloride or $\mathrm{MPO}-\mathrm{H}_{2} \mathrm{O}_{2}$-nitrite system using 2 different concentrations of $\mathrm{H}_{2} \mathrm{O}_{2}$. The rh-sFasL formed multimers when incubated with $\mathrm{MPO}, \mathrm{H}_{2} \mathrm{O}_{2}$, and $\mathrm{NaCl}$ but not when incubated with MPO- $\mathrm{H}_{2} \mathrm{O}_{2}$ or MPO-NaCl alone or with the MPO- $\mathrm{H}_{2} \mathrm{O}_{2}$ $\mathrm{NaNO}_{2}$ system (Figure $3 \mathrm{~A}$ ). The effects of $\mathrm{H}_{2} \mathrm{O}_{2}-\mathrm{MPO}-\mathrm{Cl}^{-}$or $\mathrm{HOCl}$ on sFasL multimerization were dose dependent (Figure 3, B and C), and high concentrations of $\mathrm{H}_{2} \mathrm{O}_{2}$ and MPO with $\mathrm{Cl}^{-}$, as well as $\mathrm{HOCl}$ alone, caused progressive loss of the sFasL bands, suggesting degradation or loss of reactive epitopes. Importantly, preincubation with the $\mathrm{HOCl}$ scavenger L-methionine completely prevented the multimerization of human $\mathrm{sFasL}$ as well as its degradation with the highest concentrations of $\mathrm{H}_{2} \mathrm{O}_{2}$ and $\mathrm{MPO}$ or $\mathrm{HOCl}$ (Figure 3, $\mathrm{B}$ and $\mathrm{C}$ ). Therefore, $\mathrm{MPO}$-derived oxidants, such as $\mathrm{HOCl}$, provide a mechanism for the multimerization of sFasL in the BAL fluid of patients with ARDS as well as for its degradation.

The oxidized multimers of sFasL did not dissociate in SDSPAGE with reducing agents such as $2-\mathrm{ME}$ and heat $\left(+96^{\circ} \mathrm{C}\right)$ (Figure 3, A-C) or with high concentrations of urea $(8 \mathrm{M})$ or high concentrations of dithiothreitol (DTT) (10 or $20 \mathrm{mM}$ ) (Figure 3, $\mathrm{D}$ and $\mathrm{E}$, respectively). To explore the role of oxidized methionines in multimerization of sFasL, which could have been produced by the oxidants in the prior experiments, we incubated oxidized sFasL with dimethyl sulfide (DMS) and hydrochloric acid ( $\mathrm{HCl})$, which is reported to reduce methionine sulfoxide to methionine (ref. 37 and Figure 3F). This resulted in the dissociation of aggregates of sFasL to monomers and lower molecular weight fragments. Taken together, these results suggest that methionine oxidation is involved in aggregation of sFasL through the formation of disulfide-independent intramolecular bonds. 
A

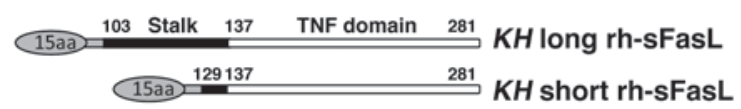

B
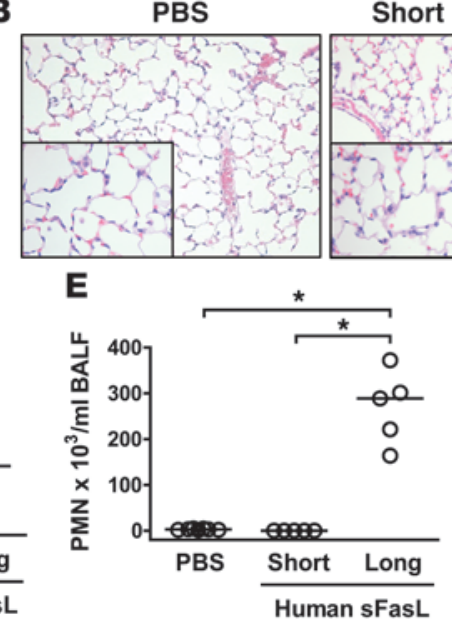

H
Short h-sFasL

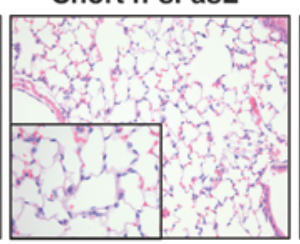

F

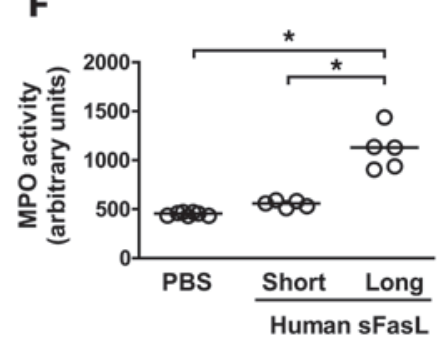

G
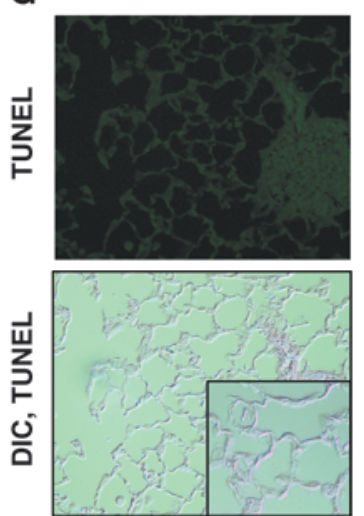

I

Cytokeratin

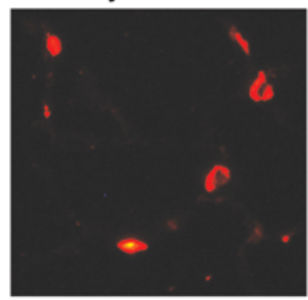

D
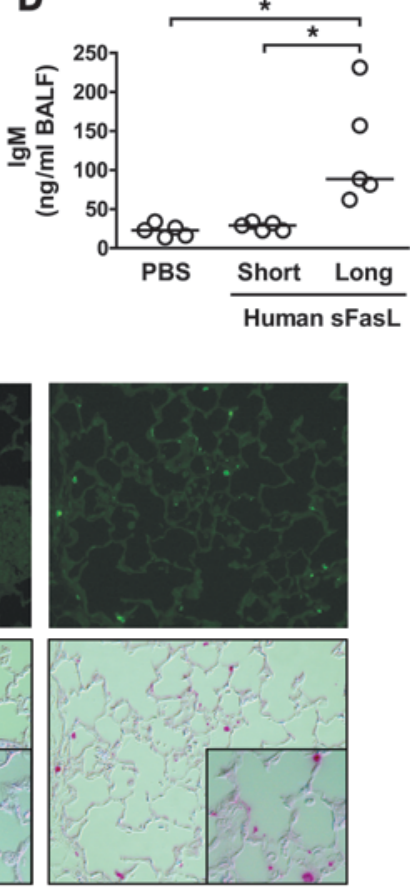

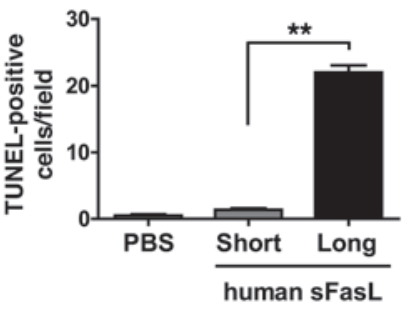

DIC

Cytokeratin (blue) TUNEL (pink)

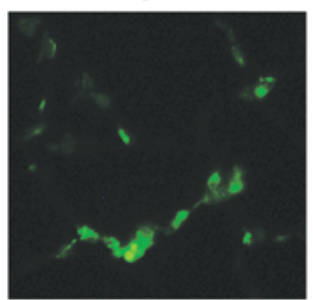

Cytokeratin
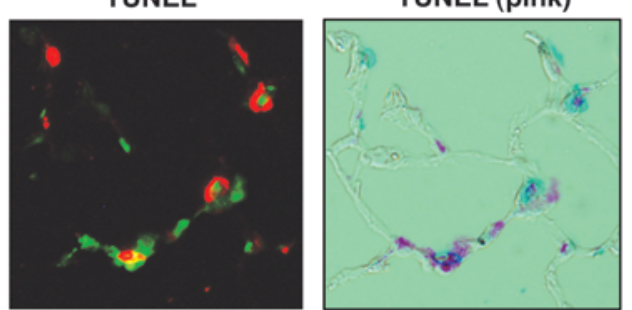

J

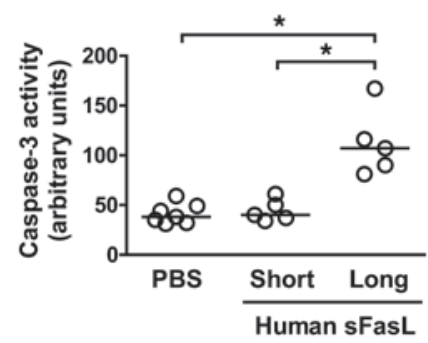

Figure 6

Effect of the stalk region at the $\mathrm{N}$ terminus on the biological activity of human sFasL in vivo. (A) Structure of the short and long variants of human sFasL produced in HEK cells. The short rh-sFasL contains only the TNF domain. Both proteins have a leading 15-amino acid linker peptide encoded by the expression vector. (B-H) Effects of long and short rh-sFasL in mouse lungs 16 hours after intratracheal instillation (25 ng/g body weight). (B) Representative lung tissue sections stained with hematoxylin and eosin, showing alveolar wall thickening, vascular congestion, alveolar hemorrhage, and neutrophilic infiltrates in mice treated with long rh-sFasL. h-sFasL, human sFasL. (C-F) Effect of long rh-sFasL on lung wet weight, concentration of IgM in BAL fluid and PMNs in BAL fluid, and MPO activity in lung homogenates. (G) Analysis of cell death in lung tissue sections (TUNEL, green dots) and the overlap of differential interference contrast (DIC) image and TUNEL fluorescence signal (DIC, TUNEL). (H) Measurement of TUNEL-positive cells in lung sections (mean \pm SD). (I) Single section of mouse lung, dually stained with the TUNEL method, followed by staining with an antibody to cytokeratin, and then visualized by fluorescence microscopy using a red wavelength (cytokeratin) or a green wavelength (TUNEL) and light microscopy (DIC). The merged image shows cells that are dual labeled by the TUNEL method and the cytokeratin antibody (epithelial cells). (J) Caspase-3 activity in lung homogenates. Each dot represents an individual mouse. Horizontal bars represent medians. ${ }^{\star} P<0.01 ;{ }^{* \star} P<0.001$. Original magnification, $\times 200$ (B, G, and I); insets $(\times 400)$. 


\section{Table 1}

Cytokine and chemokine concentrations in lung homogenates of C57BL/6 mice 16 hours after intratracheal instillation of short or long rh-sFasL

$\begin{array}{lccc} & \text { PBS } & \text { Short rh-sFasL } & \text { Long rh-sFasL } \\ \mathrm{IL}-1 \beta(\mathrm{pg} / \mathrm{ml}) & 116.4 \pm 23.2 & 103.2 \pm 16.1 & 724.2 \pm 450.7^{\mathrm{A}} \\ \mathrm{IL}-6(\mathrm{pg} / \mathrm{ml}) & 38.1 \pm 8.9 & 43.1 \pm 7.5 & 168.4 \pm 57.9^{\mathrm{A}} \\ \mathrm{IL}-4(\mathrm{pg} / \mathrm{ml}) & 144.6 \pm 23.7 & 171.5 \pm 14.6 & 100.2 \pm 6.6^{\mathrm{B}} \\ \mathrm{KC}(\mathrm{pg} / \mathrm{ml}) & 54.0 \pm 16.3 & 43.1 \pm 8.5 & 331.3 \pm 123.4^{\mathrm{A}} \\ \mathrm{MIP}-2(\mathrm{pg} / \mathrm{ml}) & 19.2 \pm 7.4 & 20.8 \pm 14.0 & 344.9 \pm 265.9^{\mathrm{A}} \\ \text { MCP-1 }(\mathrm{pg} / \mathrm{ml}) & 426.0 \pm 217.2 & 455.9 \pm 102.7 & 1,865.0 \pm 1,175.0^{\mathrm{A}}\end{array}$

Mean \pm SD. ${ }^{A} P<0.01$ vs. short rh-sFasL and PBS; ${ }^{B} P<0.05$ vs. short rh-sFasL and PBS.

ARDS BAL fluid oxidizes methionine residues in sFasL. To investigate the role of methionine and other amino acids in the aggregation of sFasL in ARDS BAL fluid, we incubated rh-sFasL in normal, at-risk, or ARDS BAL fluid and then purified the rh-sFasL using an immunoaffinity column. The recovered rh-sFasL was digested with trypsin, and the tryptic peptides were analyzed by liquid chromatography-electrospray ionization-tandem mass spectrometry analysis (LC-ESI-MS/MS). We focused on the tryptic peptide $\mathrm{Y}^{218} \mathrm{PQDLVMMEGK}$, because $\mathrm{Y}^{218}$ is in the Fas binding site, and nearby tyrosine and methionine residues are targets for modification. When sFasL was incubated with normal or at-risk BAL fluid, a major peak of material with a mass-to-charge ratio $(\mathrm{m} / \mathrm{z})$ anticipated for the doubly protonated peptide $(\mathrm{m} / \mathrm{z}, 656.1)$ was detected (Figure 4A, normal and at risk). Analysis by tandem mass spectrometry (MS/MS) confirmed that the sequence of this peptide was YPQDLVMMEGK (Figure 4B, top row). In contrast, when sFasL was incubated in BAL fluid from patients with ARDS, the $\mathrm{Y}^{218} \mathrm{PQDLVMMEGK}$ peptide almost disappeared and a major peak of new material appeared with a $\mathrm{m} / \mathrm{z}$ of 672.1 (doubly charged) (Figure 4A, ARDS), suggesting that the molecular weight of the original peptide had increased by $32 \mathrm{Da}$, corresponding to the gain of 2 oxygen atoms. The MS/MS analysis of the modified peptide revealed that each of the methionine residues in the peptide gained $16 \mathrm{Da}$, indicating that they were oxidized to methionine sulfoxide (Figure 4B, bottom row). In contrast, chlorination or nitration of $\mathrm{Y}^{218}$ were not detected, suggesting that the methionine residues of sFasL are major targets of oxidation by reactive intermediates in BAL fluid of patients with ARDS.

Methionine residues are involved in oxidation-mediated multimerization of $s$ Fas $L$. To confirm that oxidation of methionine residues is involved in the multimerization of sFasL, we took advantage of the fact that murine sFasL contains fewer methionine residues than human sFasL. We cloned the mouse sFasL cDNA and used sitedirected mutagenesis to change specific residues to methionine at the locations of methionine residues in human sFasL (Figure 5A). This "humanized" mouse sFasL contained a total of 7 methionine residues (mouse met-sFasL). When the mouse met-sFasL and the native form of mouse sFasL were subjected to mild oxidation with $\mathrm{HOCl}$, the mouse met-sFasL multimerized more readily than the native sFasL (Figure 5B), and the prior addition of L-methionine prevented multimerization (Figure 5B, lanes 1 and 5).

We then compared the biological activities of the humanized mouse met-sFasL and the mouse native sFasL using MLE-15 mouse lung epithelial cells (Figure 5C). Neither mouse native sFasL nor mouse met-sFasL induced either expression of C-X-C chemokine (KC) or activation of caspase- 3 in MLE-15 cells in nonoxidative or oxidative conditions, despite the high concentrations of both proteins tested $(800 \mathrm{ng} / \mathrm{ml})$, and cell death also was unaffected (data not shown). In contrast, a lower concentration of rh-sFasL (400 ng/ml) was biologically active, as shown by increased $\mathrm{KC}$ expression and caspase- 3 activation in MLE- 15 cells. Therefore, increasing the number of methionine residues in mouse sFasL promoted multimerization in oxidative conditions, but this by itself was not sufficient to increase the biological activity of this form of mouse sFasL.

Relevance of the $N$-terminal stalk region for bioactivity of $s F a s L$. Because human sFasL was biologically active in MLE-15 cells, whereas mouse met-sFasL was not, we explored the possibility that structural determinants other than methionine residues also affected the biological activity of sFasL. The mouse sFasL (both native and met-sFasL) that we used contained only the TNF domain at the $\mathrm{C}$ terminus, whereas the commercial form of human sFasL contained the C-terminal TNF domain as well as a stalk domain at the extracellular $\mathrm{N}$ terminus. Therefore, we expressed a long form of human sFasL containing both the stalk and the TNF domains (103-281 aa) and a short form of human sFasL containing the TNF domain and only 6 amino acids of the stalk region (129-281 aa) (Figure 6A) and then tested the biological activity of each form of sFasL in the lungs of C57BL/ 6 mice.

Lung histology, permeability, and inflammation. Intratracheal instillation of the long form of human sFasL ( $25 \mathrm{ng} / \mathrm{g}$ body weight) resulted in overt alveolar wall thickening, vascular congestion, alveolar hemorrhage, and neutrophilic infiltration, whereas the lungs of mice treated with the short form of sFasL were histologically normal (Figure 6B). At 16 hours after instillation, the wet weight of the lungs increased significantly in the mice treated with the long form of human sFasL but not in mice treated with the short form (Figure 6C). The concentration of IgM in BAL fluid, used as a marker of protein permeability, was significantly increased in mice treated with long human sFasL (Figure 6D), but was unaffected in mice treated with the short human sFasL. The long human sFasL caused a significant increase in the total number of polymorphonuclear leukocytes (PMNs) in BAL fluid, whereas the short form of human sFasL had no effect on the BAL cell profile (Figure $6 \mathrm{E}$ ).

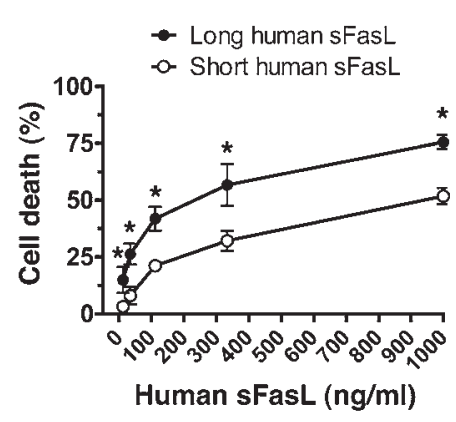

\section{Figure 7}

Cytotoxicity of long and short forms of rh-sFasL in Jurkat cells. Jurkat cells were incubated with media supplemented with serial concentrations of the long or short forms of rh-sFasL, and cytotoxicity was determined 18 hours later using the alamarBlue assay. Cells treated with medium only were used to determine $100 \%$ survival. The long human sFasL was more cytotoxic at each concentration of sFasL. Data are the mean \pm SD of 3 separate experiments performed in duplicates. ${ }^{\star} P<0.05$ for long vs. short human sFasL. 

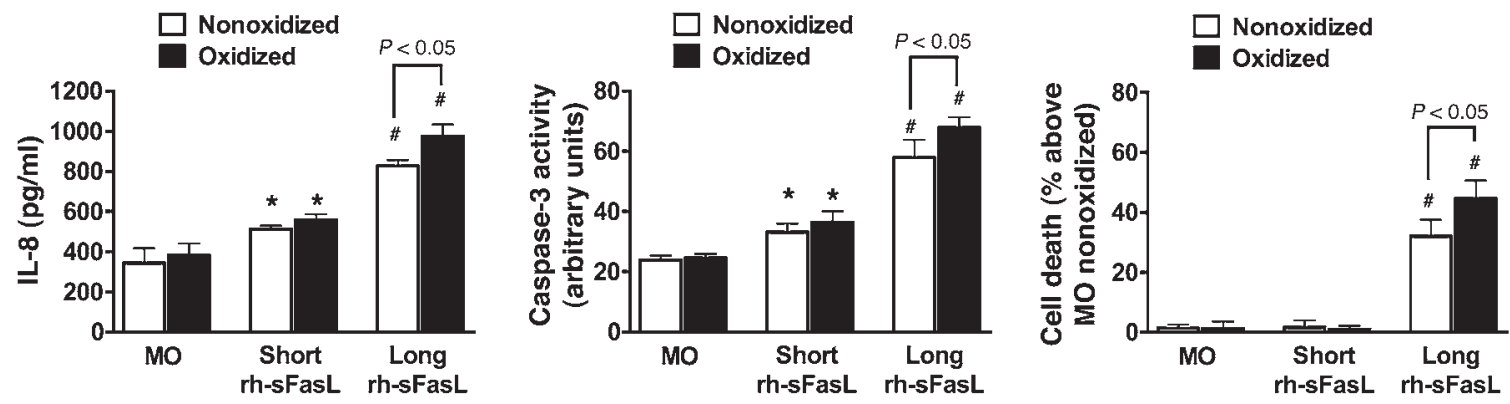

Figure 8

Effect of oxidation on the bioactivity of long and short human sFasL for human SAECs. Human SAECs were incubated with supernatants of transfected HEK cells that express either long or short rh-sFasL. These supernatants were pretreated with (black bars) or without (white bars) $\mathrm{HOCl}(100 \mu \mathrm{M})$. MO, medium only; SAECs incubated with supernatant from nontransfected HEK cells, with or without $\mathrm{HOCl}$ pretreatment. Expression of IL-8, caspase-3 activity, and cell death were determined after 18 hours by ELISA, enzymatic assay, and alamarBlue, respectively. SAECs treated with medium only were used to determine $100 \%$ survival. Long rh-sFasL was significantly more bioactive than short rh-sFasL, and oxidation enhanced the bioactivity of long rh-sFasL. Data are the mean $\pm \mathrm{SD}$ of 3 separate experiments performed in duplicate. ${ }^{\star} P<0.05$ vs. medium only; ${ }^{\#} P<0.05$ vs. medium only and short rh-sFasL.

Accordingly, the MPO activity in lung homogenates was significantly increased only in the lungs of mice treated with the long rh-sFasL (Figure 6F). The lungs of mice treated with the long human sFasL contained higher concentrations of the proinflammatory cytokines IL-1 $\beta$, IL-6, KC, MIP-2, MCP-1, and MIP-2 and lower concentrations of the antiinflammatory cytokine IL-4 (Table 1), whereas cytokine concentrations were not increased in the lungs of mice treated with the short human sFasL. TNF- $\alpha$, IFN- $\gamma$, IL-10, and IL-12 were not detected in any of the groups.
Activation of proapoptotic pathways. Analysis of lung tissue sections using the TUNEL method showed that the number of nuclei containing DNA strand breaks was significantly higher in mice treated with the long form of sFasL than in those treated with the short form $(P<0.05)$ (Figure 6G). Fluorescent double staining of DNA fragmentation and cytokeratin (marker of epithelial cells) showed that some TUNEL-positive cells in the alveolar wall were also positive for cytokeratin, indicating that alveolar epithelial cells were undergoing apoptosis (Figure 6I). Some cells were TUNEL positive but cytokeratin neg-
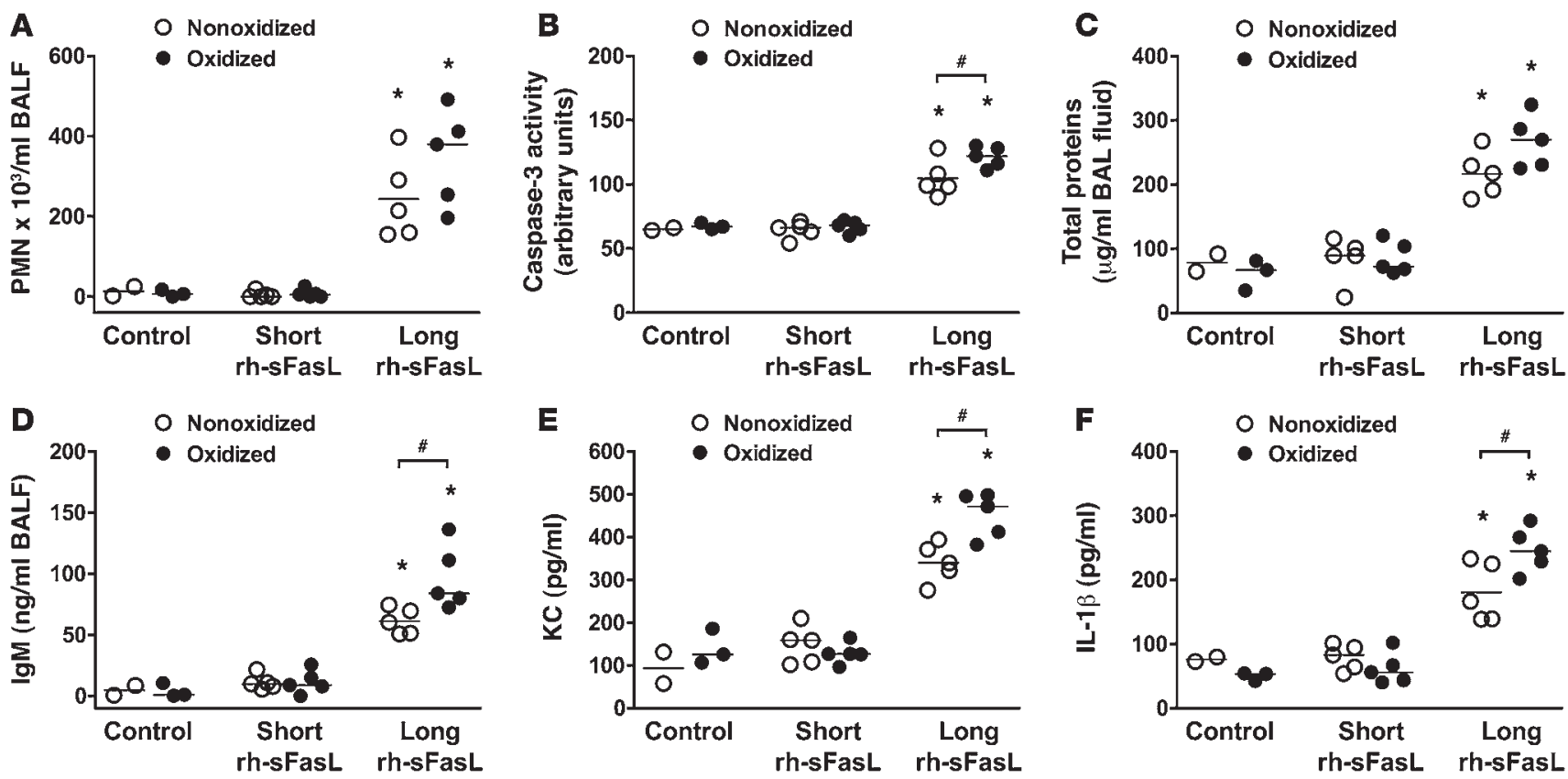

Figure 9

Effect of oxidation on the bioactivity of long and short rh-sFasL in mouse lungs in vivo. Mice were treated with 1 intratracheal instillation of HEK cell supernatant, containing short or long human sFasL, and pretreated with or without $\mathrm{HOCl}$ as an oxidant and studied 16 hours later. Control refers to nontransfected HEK cell supernatant pretreated with or without $\mathrm{HOCl}(100 \mu \mathrm{M})$. The oxidized long rh-sFas caused greater increases in (A) the number of PMNs in BAL fluid, (B) caspase-3 activation in lung homogenates, (C and $\mathbf{D})$ concentration of total protein and IgM in BAL fluid, and (E and $\mathbf{F})$ expression of $\mathrm{KC}$ and IL-1 $\beta$ in lung homogenates. Each dot represents a single mouse. Horizontal bars represent medians. ${ }^{*} P<0.05$ vs. control supernatant and short rh-sFasL; ${ }^{*} P<0.05$. 

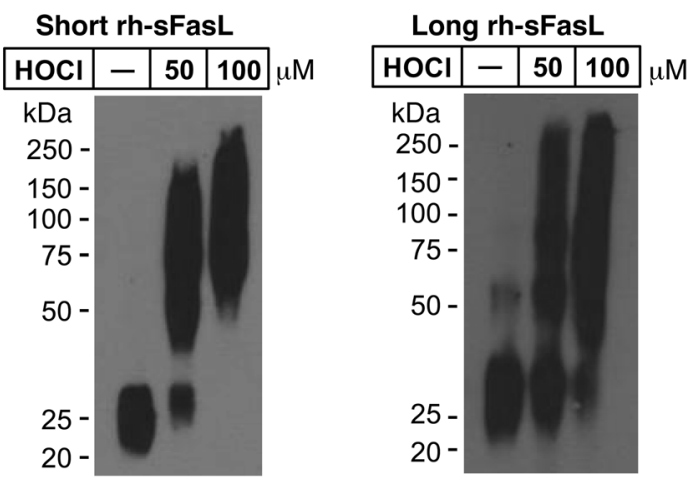

Figure 10

Oxidation-mediated multimerization of short and long sFasL. Western blot analyses of short and long rh-sFasL incubated in PBS alone or in the presence of 2 different concentrations of $\mathrm{HOCl}(\mu \mathrm{M})$. After 15 minutes, the reaction was terminated with $2.5 \mathrm{nM} \mathrm{L-methionine.} \mathrm{Both} \mathrm{short}$ and long human sFasL have the same aggregation status in basal conditions. Following oxidation with $\mathrm{HOCl}$, short and long rh-sFasL showed similar multimerization $(50-\mathrm{kDa}$ and larger molecular weight bands). Western blot analyses were performed using SDS-PAGE with reducing conditions (2-ME and heat).

ative, indicating that other populations of lung cells were undergoing apoptosis. Caspase- 3 activity was significantly increased only in the lungs of mice treated with long human sFasL (Figure 6J).

All of the alterations observed in the lungs of mice treated with intratracheal instillation of the long form of sFasL were absent in lpr mice, which carry a nonfunctional Fas receptor, indicating that the long human sFasL causes lung injury and inflammation by activating the murine Fas receptor (data not shown).

The stalk region and oxidation of human sFasL influence bioactivity in buman cells. To test whether the stalk region of human sFasL also influences biological activity in human cells, we treated human Jurkat cells with the long and short forms of human sFasL (Figure 7). The long human sFasL was cytotoxic at a concentration of $12.3 \mathrm{ng} / \mathrm{ml}$ $(15 \% \pm 5.6 \%)$, whereas the dose response of the short form of human sFasL was shifted significantly to the right (onset of cytotoxicity at $111.1 \mathrm{ng} / \mathrm{ml})$. On a molar basis, this difference in cytotoxicity is even more pronounced.
To test whether oxidation modifies the biological activity of the long and short forms of human sFasL in human lung epithelial cells, we transfected human embryonic kidney (HEK) cells with cDNA directing the expression of long or short human sFasL. The supernatants of the transfected HEK cells were treated with $\mathrm{HOCl}(100 \mu \mathrm{M})$ and then incubated with human SAECs for 16 hours at $50 \%$ final concentration (Figure 8). Compared with short human sFasL, exposure of SAECs to long human sFasL induced higher expression of IL-8 (511.8 $\pm 17.6 \mathrm{pg} / \mathrm{ml}$ vs. $826.3 \pm 28.7 \mathrm{pg} / \mathrm{ml} ; P<0.05)$, caspase-3 activity (33.2 \pm 2.9 vs. $57.8 \pm 5.9$ arbitrary units; $P<0.05)$ and percentage of cell death $(1.7 \% \pm 2.3 \%$ vs. $32.0 \% \pm 5.5 \% ; P<0.05)$. Oxidation increased the biological activity of long human sFasL but did not change the bioactivity of short human sFasL.

The stalk region and oxidation of human sFasL influence bioactivity in mouse lungs. To determine whether oxidation modifies the biological activity of the long and short forms of human sFasL in the lungs in vivo, we treated mice with a single intratracheal instillation of HEK cell supernatant containing long human sFasL (nonoxidized or oxidized) or short human sFasL (nonoxidized or oxidized) (Figure 9). In all cases, the long form was more bioactive than the short sFasL (Figure 9). Oxidation increased the bioactivity of the long form of human sFasL, as shown by an increase in the number of PMNs in BAL fluid, increased caspase-3 activity in lung homogenates, higher concentrations of IgM in the BAL fluid, and higher expression of KC and IL-1 $\beta$ in lung homogenates, although not all the responses reached statistical significance. In contrast, oxidation did not increase the bioactivity of the short form of human sFasL. Thus, the stalk region is a critical determinant of the biological activity of human sFasL and oxidation enhances the activity of the long rh-sFasL in vivo.

Long and short human sFasL have similar capacity to aggregate. In order to determine whether the stalk region could cause differences in the degree of aggregation of sFasL, we compared the aggregation status of recombinant short and long human sFasL in basal or oxidative conditions (Figure 10). In nonoxidative conditions, both short and long human sFasL appeared as monomers $(25 \mathrm{kDa})$. After oxidation with $\mathrm{HOCl}$, both short and long human sFasL showed similar degrees of dose-dependent aggregation, suggesting that the stalk region in sFasL does not enhance aggregation, even when the protein is oxidized.

\section{Structural domains of human FasL}

\section{Figure 11}

Primary structure of FasL. The mFasL is composed of intracellular (1-80 aa), transmembrane (81-102 aa), and extracellular (103-281 aa) domains. The extracellular domain contains the stalk region at the $\mathrm{N}$ terminus and the TNF homology domain (THD) at the $\mathrm{C}$ terminus. The amino acid sequence of the extracellular domain of FasL is shown. The stalk region is underlined, and the TNF homology domain region is italicized. Two cleavage sites for MMP-7 (1st, ELAEL; 2nd, SL) are present in the stalk region of the extracellular domain of FasL. Seven methionines (bold) are present in the TNF homology domain, and one is in the stalk region.

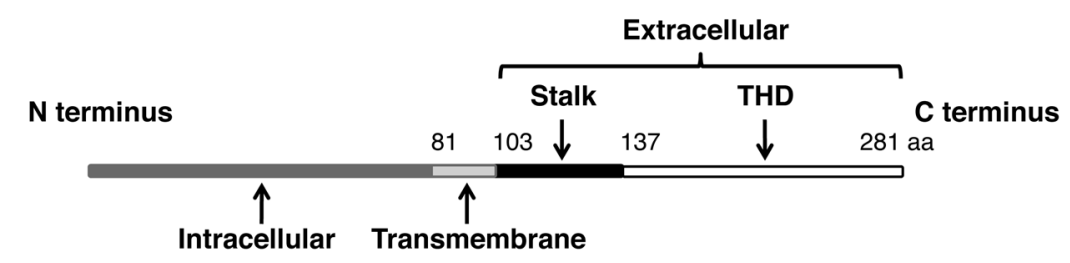

Amino acid sequence of the extracellular domain of human FasL OLFHLOKELAELRESTSOM ${ }^{121}$ 2nTASTLEKQIGHPSPPPEKKELRKVAHLTGKSNSRSM $^{158}$ PLEWEDTYGIVLLSGVKYKKGGLVINETGLYFVYSKVYFRGQSCNNLPLSHKVYM ${ }^{213}$ RNSK YPQDLV $\mathrm{M}^{224} \mathrm{M}^{225}{ }_{\text {EGKM }} \mathrm{M}^{229} \mathrm{M}^{230}$ SYCTTGQM ${ }^{238}$ WARSSYLGAVFNLTSADHLYVNVSELSLV NFEESQTFFGLYKL 281 
A

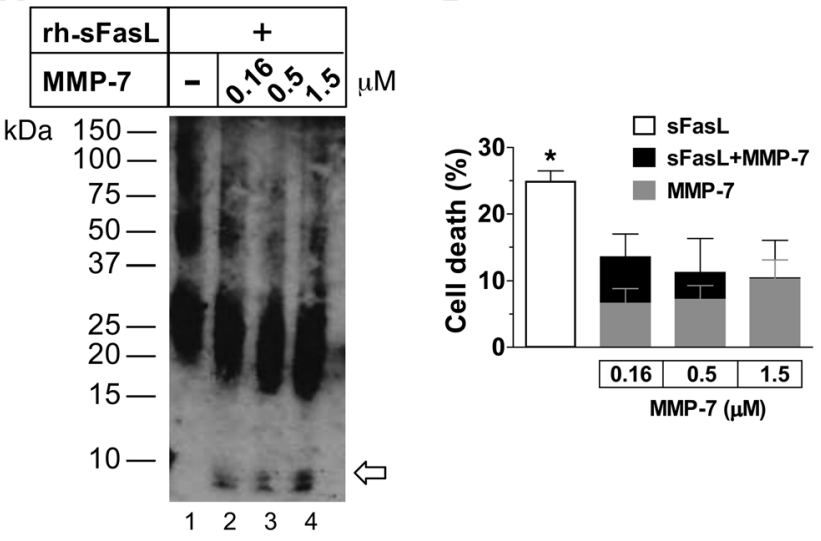

Figure 12

Effect of MMP-7 on structure and cytotoxicity of human sFasL. (A) Incubation of the long form of the rh-sFasL $(0.5 \mu \mathrm{M})$ with serial concentrations of MMP-7 $(0.16,0.5$, or $1.5 \mu \mathrm{M})$. The samples were analyzed by Western blot using SDS-PAGE in nonreducing conditions using a polyclonal antibody against human SFasL and (B) tested for cytotoxicity in Jurkat cells. (A) Treatment with serial concentrations of MMP-7 (lanes $2-4)$ resulted in a shift of the monomers $(25 \mathrm{kDa})$ of sFasL to a lower molecular weight and the appearance of a new band below $10 \mathrm{kDa}$ (arrow) corresponding to the predicted molecular weight of the stalk region plus the 15-amino acid linker peptide encoded by the expression vector. Numbers on the left show molecular weight. (B) Cytotoxicity of native and MMP-7-treated rh-sFasL in Jurkat cells at 18 hours using the alamarBlue assay. The white bar shows the cytotoxicity of untreated SFasL; the gray bars show the cytotoxicity of MMP-7 alone. Incubation with MMP-7 reduced the cytotoxicity of long human SFasL (black bars) as compared with the effect of MMP-7 alone (gray bars). The data are mean \pm SD of 3 separate experiments performed in duplicate. ${ }^{*} P<0.05$ vs. long human SFasL and MMP-7 alone.

Proteolytic cleavage of the stalk region by MMP-7 reduces the biological activity of buman sFasL in vitro. The recombinant long human $\mathrm{SFasL}$ contains 2 MMP-7 cleavage sites near the $\mathrm{N}$ terminus, 1 near the cell membrane and 1 near the beginning of the TNF homology domain (Figure 11). We hypothesized that cleavage of the long sFasL protein at the Ser126-Leu127 (S-L) site by MMP-7 would release the stalk region, transforming the active long sFasL protein into the less active short sFasL. To test this, affinity-purified long human sFasL, derived from supernatant of transfected HEK cells, was incubated with different concentrations of human MMP-7 (Figure 12A). Whereas the long human sFasL showed a prominent band at $25 \mathrm{kDa}$, corresponding to monomeric sFasL, treatment with MMP-7 shifted the monomeric band to a lower molecular weight, and a new band below $10 \mathrm{KDa}$ appeared, corresponding to the predicted molecular weight of the stalk region with the extra 15-amino acid linker peptide from the expression vector (Figure 12A). Importantly, treatment of sFasL with MMP-7 led to a significant reduction of cytotoxicity for Jurkat cells (Figure 12B). We further used nanoLC-ESI-MS/MS to detect cleavage of sFasL by MMP-7. The long sFasL proteins, either treated or untreated with MMP-7, were digested with trypsin, and the tryptic peptides were analyzed using nanoLC-ESI-MS/MS (Figure 13). MMP-7 cleaved long human SFasL at the S-L site (about $40 \%$ of protein, Figure 13A), resulting in the release of the ESTSQM ${ }^{121}$ HTASS peptide in the stalk region. Together, these results show that MMP-7 cleaves the stalk region of sFasL and reduces the biological activity of the long human sFasL in vitro. Interestingly, oxidation of $\mathrm{Met}^{121}$ in the stalk region of the sFasL prevented cleavage at the S-L site by MMP-7 (Figure 13B), suggesting that oxidation of methionine residues could be a mechanism that prevents cleavage of the stalk region of human sFasL in vivo.

The long form of sFasL is present in the BAL fluid of patients with ARDS and contains oxidized methionine residues. To determine whether $\mathrm{SFasL}$ released into the airspaces of patients with ARDS contains the stalk region and whether its methionine residues are oxidized, we analyzed the structure of sFasL in the BAL fluid of patients with ARDS. Human ARDS BAL fluids were pooled, the endogenous sFasL was immunoaffinity purified and digested with trypsin, and the tryptic peptides were analyzed using nanoLC-ESI-MS/MS. We focused on the peptide ESTSQM ${ }^{121}$ HTASSLEK in the stalk region, which contains the S-L MMP-7 cleavage site (Figure 11). Analysis using nanoLC-ESI-MS/MS with specific reaction monitoring detected the oxidized peptide ESTSQM $(\mathrm{O}){ }^{121} \mathrm{HTASSLEK}$ $(\mathrm{m} / \mathrm{z}, 776.5$, doubly charged) but almost none of the nonoxidized form ESTSQMTASSLEK (m/z, 768.5, doubly charged) (Figure 14). This indicates that human sFasL present in the ARDS BAL fluid corresponds to a long form containing the stalk region at the $\mathrm{N}$ terminus, in which methionine is oxidized.

For comparison, when recombinant sFasL was incubated with MPO- $\mathrm{H}_{2} \mathrm{O}_{2}-\mathrm{Cl}^{-}$for 1 hour, $99 \%$ of the methionine residues in the stalk region were oxidized (ESTSQM $[\mathrm{O}]^{121} \mathrm{HTASSLEK}$ ) and $70 \%$ of the peptides containing the receptor binding site were either singly $\left(41.8 \%\right.$, YPQDLVM $\left.[\mathrm{O}]^{224} \mathrm{M}^{225} \mathrm{EGK}\right)$ or doubly oxidized $(29.9 \%$, YPQDLVM[O $\left.]^{224} \mathrm{M}[\mathrm{O}]^{225} \mathrm{EGK}\right)$ at methionine residues. When recombinant sFasL was diluted in PBS, incubated for 1 hour, and analyzed by the same MS/MS procedures, less than $1.5 \%$ of the methionine residues in the stalk peptide (ESTSQM ${ }^{121}$ HTASSLEK) were oxidized and none of the methionine residues near the receptor-ligand binding site were oxidized (YPQDLVM ${ }^{224} \mathrm{M}^{225} \mathrm{EGK}$ ), suggesting that spontaneous oxidation is uncommon.

Taken together, the results suggest the following hierarchical order of biological activity of the various forms of human sFasL: oxidized long human sFasL > nonoxidized long sFasL > oxidized short sFasL and nonoxidized short sFasL > MMP-7-cleaved sFasL, which could have equal or lesser cytotoxicity than short sFasL, depending on the extent of cleavage.

\section{Discussion}

The main goal of this study was to determine the structural factors that modulate the biological activity of human sFasL in the lungs of patients with lung injury. Our results indicate that the endogenous sFasL released into the airspaces of patients with ARDS is highly aggregated and contains both oxidized methionine residues and the $\mathrm{N}$-terminal stalk region. We show for the first time to our knowledge that the stalk region in the $\mathrm{N}$ terminus is a critical determinant of the biological activity of sFasL in vitro and in vivo. In addition, oxidants present in ARDS BAL fluid promote aggregation of sFasL by mechanisms involving oxidation of methionine residues. This oxidation-mediated aggregation of sFasL increases the bioactivity of sFasL in mouse lungs and in human lung epithelial cells. However, oxidation does not modify the bioactivity of the short form of sFasL lacking the stalk region, suggesting that the stalk region is required for full biological activity. Moreover, cleavage of the stalk region by the proteolytic action of MMP-7 reduces the biological activity of sFasL, and oxidation of the stalk region prevents MMP-7-mediated cleavage of sFasL. 

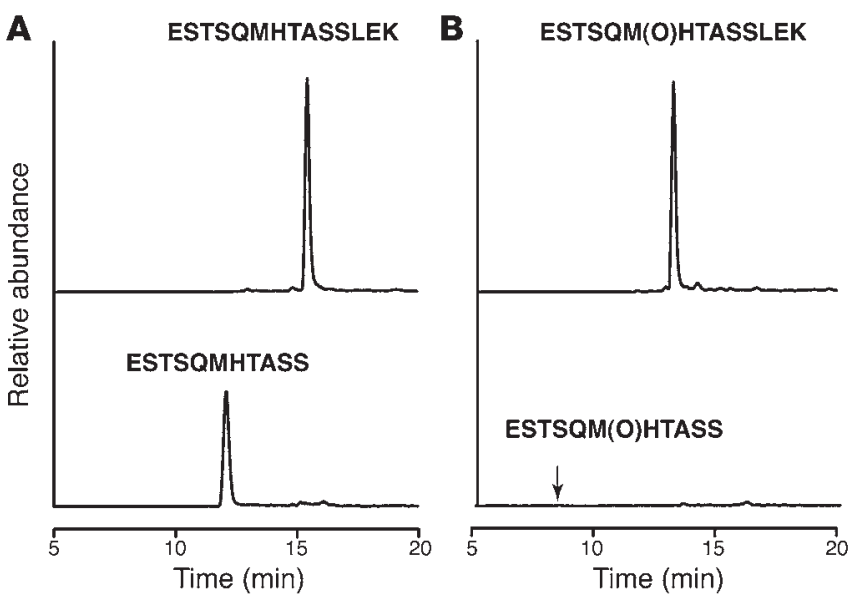

Figure 13

Effect of oxidation of Met ${ }^{121}$ on cleavage of rh-sFasL by MMP-7. rh-sFasL was incubated with MMP-7 $(0.25 \mu \mathrm{M})$ as in Figure 12, then digested with trypsin and analyzed using nanoLC-MS. (A) Reconstructed ion chromatogram of uncleaved nonoxidized peptide ESTSQM ${ }^{121} \mathrm{HTASSLEK}$ ( $\mathrm{m} / \mathrm{z} 768.5$, doubly charged) and its cleaved product ESTSQM ${ }^{121} \mathrm{HTASS}$ (m/z 583.3, doubly charged), indicating that approximately $40 \%$ of the nonoxidized peptide was cleaved as detected by peak area. (B) Reconstructed ion chromatogram of uncleaved oxidized peptide ESTSQM $(O)^{121} \mathrm{HTASSLEK}(\mathrm{m} / \mathrm{z} 776.5$, doubly charged) and its cleaved product ESTSQM $(\mathrm{O})^{121} \mathrm{HTASS}$ ( $\mathrm{m} / \mathrm{z} 591.3$, doubly charged), indicating that only $0.5 \%$ of the oxidized peptide was cleaved.

A number of studies have provided evidence supporting the role of the Fas/FasL system in the pathogenesis of lung injury $(11,26$, $38-41)$. In the lungs of patients with ARDS, there is an increase in the expression of Fas and FasL in the alveolar walls associated with activation of apoptotic pathways $(26,38)$. In the current study, we have confirmed our previous observation that the sFasL is elevated in the BAL fluid of patients at the onset of ARDS and induces apoptosis in primary human lung epithelial cells in vitro (26). In this study, activation of Fas pathway in mouse lungs by intratracheal instillation of human sFasL caused lung injury, with disruption of the alveolar epithelial barrier and apoptosis of cells in the alveolar wall. Similar damage also has been found in models of lung injury caused by intratracheal instillation of Fas-activating antibody (in mouse lungs) or human sFasL (in rabbit lungs) $(10,11)$. The importance of the Fas pathway also has been shown in other models of lung injury caused by direct insults, such as LPS, hypoxia-induced lung injury during Legionella pneumonia, or IgG immune complexes (42-44), and systemic insults, such as hemorrhagic shock combined with cecal ligation and puncture (45).

Apoptosis of alveolar epithelial cells is one of the mechanisms that are likely to contribute to the disruption of the alveolar epithelial barrier in ARDS $(9,11,26,39-42)$. Our observation that alveolar epithelial cells undergo apoptosis in mouse lungs after instillation of human sFasL is consistent with the studies of Fine and colleagues, showing that activation of Fas by the intratracheal administration of a Fas-activating $\mathrm{mAb}(\mathrm{Jo} 2)$ results in apoptosis of murine alveolar type II pneumocytes in vivo (46). Apoptosis of type II pneumocytes is also involved in repair of alveolar epithelial damage. Proliferation of type II pneumocytes occurs in the early phase of ALI and is important for repair of alveolar wall damage. Fas-dependent apoptosis of proliferating type II pneumocytes has been implicated in the disappearance of these cells in the resolution phase (5). Therefore, at the onset of ALI, activation of sFasL can contribute to the death of alveolar epithelial cells, but later in the course of lung injury, this pathway is likely to contribute to repair.

The mechanism by which the stalk region controls the biological activity of sFasL remains uncertain. The stalk region, even under oxidative conditions, does not increase the ability of human sFasL to aggregate and, therefore, this mechanism is unlikely to explain the increased biological activity of the long form of sFasL. Instead, the stalk region might confer a spatial conformation that increases affinity for Fas receptor binding, or it might stabilize aggregates of sFasL, for example, by stabilizing the TNF domain structure to enhance receptor binding. Another possibility is that the stalk region might promote secondary aggregation of the FasL/Fas receptor complexes on the cell surface needed for efficient activation of Fas-dependent intracellular signaling (22). In this regard, new preliminary experiments in our laboratory show that the long human sFasL causes more rapid clustering of the Fas receptors on the surface of Jurkat cells as compared with the short form lacking the stalk region (data not shown).

MMPs have been identified in the BAL fluid of patients with ALI (47), and MMP-7 is detectable in the BAL fluid of patients with ARDS (X. Fu, unpublished observations). Human sFasL contains several cleavage sites for MMP-7, one of which is located in the stalk region (20) (Figure 11). We found that cleavage at the stalk region site reduces the biological activity of sFasL in vitro and that long sFasL containing the stalk region is present in the airspaces of patients with ARDS. Because the endogenous sFasL was fragmented by trypsin for the mass spectrometry analysis, we could not determine whether MMP-7-cleaved fragments of sFasL exist in the lung fluid of patients with ARDS or whether forms of sFasL lacking the stalk region are also present in the BAL fluid of patients with ARDS. Therefore, the relevance of MMP-7 in limiting the biological activity of sFasL in patients with lung injury requires further studies.

Protein oxidation and nitration are prominent in the lungs of patients with ALI and can modify protein function in the lung (27, $28,30,31,34)$. Therefore, we studied the effects of oxidation and nitration on the structure and bioactivity of sFasL. We focused on the oxidative and nitrosative reactions mediated by MPO and $\mathrm{H}_{2} \mathrm{O}_{2}$, as these 2 factors are mainly present in neutrophils and are abundant in the lungs of patients with ARDS. MPO- $\mathrm{H}_{2} \mathrm{O}_{2}$ in the presence of $\mathrm{NaCl}$ generates $\mathrm{HOCl}$, leading to protein oxidation, whereas MPO- $\mathrm{H}_{2} \mathrm{O}_{2}$ in the presence of $\mathrm{NO}$ generates peroxynitrite, leading to protein nitration. We found that oxidation, but not nitration, changed the structure of sFasL by promoting aggregation and oxidized sFasL at methionine residues. Methionine oxidation and increased aggregation of sFasL also occurred when exogenous sFasL was incubated in BAL fluid from patients with ARDS, but not when sFasL was incubated in BAL fluid from patients at risk for ARDS or normal volunteers, consistent with the oxidative environment in ARDS lungs. In addition, the endogenous sFasL recovered from the airspaces of patients with ARDS contained oxidized methionine residues. Methionine oxidation promoted aggregation of sFasL in vitro and was associated with an increase in the biological activity of sFasL in vitro and in vivo. Therefore, oxidation-mediated aggregation of sFasL is likely to be one of the mechanisms that affect the bioactivity of sFasL in the lungs of patients with lung injury. Oxidation, however, can have a dual role in regulating the activity of sFasL in vivo, as intense 
A

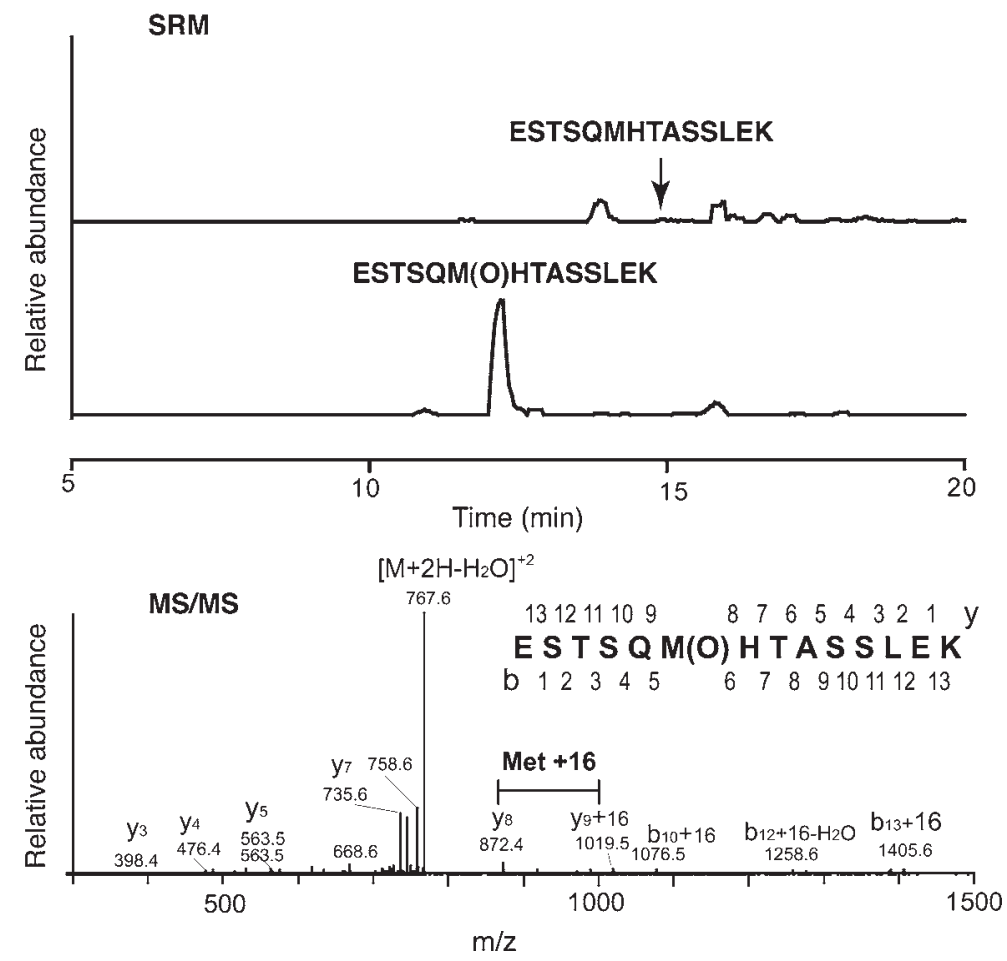

Figure 14

Oxidation of Met ${ }^{121}$ in endogenous sFasL in BAL fluid from ARDS patients. Endogenous sFasL was immunoaffinity purified from pooled BAL fluid from patients with ARDS and digested by trypsin. The tryptic peptides were analyzed using nanoLC-ESI-MS/MS. (A) Selected reaction monitoring (SRM) of ESTSQM ${ }^{121} \mathrm{HTASSLEK}(\mathrm{m} / \mathrm{z} 768.5 \rightarrow 872.4$, top tracing) and ESTSQM $(\mathrm{O})^{121}$ HTASSLEK $(\mathrm{m} / \mathrm{z} 776.5 \rightarrow 872.4$, bottom tracing). (B) MS/MS of oxidized peptide ( $\mathrm{m} / \mathrm{z} 776.5)$, showing methionine oxidation.

oxidation by the MPO- $\mathrm{H}_{2} \mathrm{O}_{2}-\mathrm{Cl}^{-}$reaction resulted in degradation of sFasL. As many other proteins are also oxidized in the BAL fluid of patients with ARDS, it is unlikely that HOCl-mediated oxidation is unique for sFasL. Likewise, it is unlikely that MPO-derived $\mathrm{HOCl}$ is the only oxidant that can affect sFasL in vivo.

The mechanisms by which methionine oxidation increases aggregation and modifies the biological activity of sFasL are still unclear. The sulfur atom of methionine is one of the most HOClreactive moieties of the 20 common amino acids. Methionine is a hydrophobic amino acid whose side chain is flexible. When oxidized, methionines become less hydrophobic and the side chain becomes more rigid. This can cause conformational changes in the tertiary structure of proteins that can lead to important changes in bioactivity, particularly when oxidation occurs in methionine residues located in or near binding sites $(33,48-51)$. These changes can modify protein-protein interactions, which could explain why methionine oxidation of long sFasL was not cleaved by MMP-7, suggesting that methionine oxidation could also have a role in preventing cleavage of sFasL by MMPs in injured lungs. Methionine oxidation also promotes aggregation of some proteins, such as human insulin-like growth factor I, in which the formation of covalent bonds is involved (52). We observed that the oxidized multimers of sFasL dissociated to monomers with DMS plus $\mathrm{HCl}$, which is reported to reduce methionine sulfoxide to methionine (37), but not with reducing agents such as $2-\mathrm{ME}$ and heat $\left(+96^{\circ} \mathrm{C}\right)$ or DTT, or urea, which disrupts noncovalent bonds (Figure 3).
These findings suggest that methionine oxidation aggregates $s F a s L$ by mechanisms involving the formation of new intermolecular bonds, which are disulfide independent.

Aggregation seems to influence the biological activity of sFasL in vitro $(22,24,25)$. We found that sFasL formed aggregates in the lung fluid of patients with ARDS and that the high molecular weight aggregates were the most cytotoxic. As suggested by others, it is possible that highly aggregated sFasL promotes more efficient aggregation of Fas receptors required for intracellular signaling (53-55). In addition, aggregation could prevent or alter the interaction of sFasL with its inhibitors, such as soluble Fas receptor (56) and Fas decoy receptor DCR3 (57), present in the alveolar fluid of patients with ALI. The data suggest that aggregation of sFasL is an important factor controlling the biological activity of sFasL; however, aggregation is not sufficient to explain the biological activity of sFasL, as aggregated forms of sFasL lacking the stalk region were not biologically active in mouse lungs.

The cytotoxicity of the BAL fluid of patients with ARDS was significantly reduced by blockade of the Fas receptor on target cells or by immunodepletion of sFasL from BAL fluid, indicating that ARDS BAL fluid induces cell death predominantly by mechanisms involving activation of the Fas/FasL pathway. Nevertheless, ARDS BAL fluid immunodepleted of sFasL also induced cell death by Fas-independent pathways, indicating that Fas/ FasL is not the only cell death pathway in ALI. This supports the complexity of lung injury, in which multiple pathways are activated simultaneously.

We conclude that the alveolar microenvironment of patients with ARDS contains factors that modulate the structure of $s$ FasL and influence its bioactivity. The net bioactivity of sFasL in the lungs of patients with ALI is likely to depend on the extent of methionine oxidation and multimerization, the degree of cleavage by MMP-7, and the presence or absence of the stalk region at the $\mathrm{N}$ terminus. These mechanisms which we believe to be novel provide a better understanding of the structural and environmental factors that govern the bioactivity of sFasL in vivo. They also provide a basis for further studies designed to determine whether inhibition of the Fas/FasL pathway is beneficial or harmful in ALI as well as other diseases in which activation of the Fas/FasL pathway is involved.

\section{Methods}

\section{Reagents}

rh-sFasL (Peprotech Inc. and Axxora-Alexis Co.) was used for in vitro and in vivo experiments, respectively. For immunoblotting of human FasL, we used the following reagents: goat anti-human FasL polyclonal Ab (Peprotech Inc.), biotinylated by using the Solid-Phase Biotinylation Kit (Pierce Biotechnology); mouse anti-human FasL mAb (BD Biosciences); HRPconjugated AffiniPure $\mathrm{F}\left(\mathrm{ab}^{\prime}\right) 2$ fragment donkey anti-mouse $\mathrm{Ab}$ (Jackson ImmunoResearch Inc.); rat anti-mouse FasL Ab (R\&D Systems); and HRP goat anti-rat $\mathrm{Ab}$ (Amersham). Neutralizing mouse anti-human Fas Ab (ZB-4) was purchased from Upstate USA Inc. and isotype-matched control 


\section{Table 2}

Characteristics of the patient population from which BAL fluids were obtained

\begin{tabular}{|c|c|c|}
\hline Patient characteristics & At risk & ARDS \\
\hline$n$ & 8 & 11 \\
\hline Age (yr) & $40.9 \pm 10.8$ & $38.1 \pm 14.2$ \\
\hline Sex (female/male) & $2 / 6$ & $5 / 6$ \\
\hline \multicolumn{3}{|l|}{ Primary risk } \\
\hline Sepsis & 4 & 2 \\
\hline Trauma & 4 & 6 \\
\hline Other & 0 & 3 \\
\hline APACHE II score ${ }^{A}$ & $25.0 \pm 2.2$ & $18.7 \pm 5.1$ \\
\hline Day of $B A L^{B}$ & Day 1 & Day 3 \\
\hline BAL fluid sFasL (pg/ml) & $30.77 \pm 53.5$ & $160.13 \pm 210.6$ \\
\hline
\end{tabular}

Age values are mean $\pm S D$. ${ }^{A}$ Mean $\pm S D$ at ICU admission. ${ }^{B}$ Day after onset of risk for ARDS or ARDS. Specific criteria for sepsis and trauma risks and for ARDS have been described by Matute-Bello et al. (58).

IgG1 was obtained from BD Biosciences. Human metalloproteinase-7 was obtained from Calbiochem. Unless otherwise indicated, all other chemicals were purchased from Sigma-Aldrich.

\section{Cloning of buman and mouse sFasL cDNA and site-directed mutagenesis}

Different lengths of human and mouse sFasL cDNA sequences were amplified in a standard PCR with DNA polymerase (Easy-A High-Fidelity PCR Cloning Enzyme, Stratagene), using cloned human FasL cDNA (catalog 10659567, ATCC) or cloned mouse FasL cDNA (catalog 10471123, ATCC) as templates. The sequences of PCR primers used for CDNA amplification were as follows: human long sFasL, sense 5'-CAGCTCTTCCACCTACAGAAGGAGC-3' and anti-sense 5'-GTCCCCAAAACATCTCTCTTG-3'; human short sFasL, sense 5'-AAGCAAATAGGCCACCCCAG-3' and anti-sense 5'-AGTTTCACCGATGGCTCAGG-3'; and mouse short sFasL, sense 5'-CCCAGTACACCCTCTGAAAAAAAAGAGCCG-3' and anti-sense $5^{\prime}$-GGCTCATGATGCAGGCATTAAGGACC- $3^{\prime}$. The PCR conditions were $95^{\circ} \mathrm{C}$ for 2 minutes; $95^{\circ} \mathrm{C}$ for 40 seconds; $55^{\circ} \mathrm{C}$ for 30 seconds and $72^{\circ} \mathrm{C}$ for 1 minute for 35 cycles; and $72^{\circ} \mathrm{C}$ for 7 minutes. The amplified sFasL cDNA was cloned into a pSecTag/FRT/V5-His-TOPO mammalian expression vector (Invitrogen). Mouse sFasL cDNA was mutated by site-directed mutagenesis in order to generate a humanized mouse sFasL using the mammalian expression vector encoding cloned native mouse sFasL cDNA as a template. Point mutations in a total of 5 codons in the plasmid encoding mouse sFasL cDNA were performed by using a QuikChange II Site-Directed Mutagenesis Kit (Stratagene), with the following mutagenic primers: sense 5'-GTATCCTGAGGATCTGGTGATGAGGAGGAGAAGATGATG-3' and anti-sense 5'-CATCATCTTCTCCTCCATCATCACCAGATCCTCAGGATAC-3' for codon 91, sense 5'-CTGCACTACTGGACAGATGTGGGCCCACAGCAGCCACCTG-3' and anti-sense $5^{\prime}$-CAGGTGGCTGCTGTGGGCCCACATCTGTCCAGTAGTGCAG-3' for codon 105, sense 5'-GCTAATGGAGGAGAAGATGATGAACTACTGCACTACTGG-3' and anti-sense 5'-CCAGTAGTGCAGTAGTTCATCATCTTCTCCTCCATTAGC-3' for codons 96 and 97, and sense 5'-GAACCCCCACTCAAGGTCCATGCCTCTGGAATGGGAAGAC- ${ }^{\prime}$ ' and anti-sense and $5^{\prime}$-GTCTTCCCATTCCAGAGGCATGGACCTTGAGTGGGGGTTC-3' for codon 25. The sequences of the cloned pSecTag/cDNA-FasL plasmids were confirmed using the Big Dye Terminator v3.1 Cycle Sequencing Kit (Applied Biosystems).

\section{Expression of sFasL protein in mammalian cells}

sFasL was expressed in HEK cells (FreeStyle 293-F cells, Invitrogen) transfected with pSecTag/cDNA-FasL encoding the human or mouse FasL cDNA using lipofection and incubated in a humidified incubator at $8 \%$ $\mathrm{CO}_{2}, 37^{\circ} \mathrm{C}$, with FreeStyle293 Expression serum-free medium (Gibco), with constant shaking. After 6 days of incubation, the cell supernatant was harvested, filtered, and stored at $-20^{\circ} \mathrm{C}$ until used. The expressed sFasL protein was purified using immunoaffinity columns (HiTrap NHS-activated HP, GE Healthcare), which were prepared according to the manufacturer's instructions, using a mouse monoclonal anti-human FasL antibody or a rat anti-mouse FasL antibody (R\&D Systems). The expressed recombinant sFasL was tested for endotoxin ( $<0.1 \mathrm{EU}$ per $1 \mu \mathrm{g}$ sFasL, measured by the Limulus Amebocyte Lysate, Pyrochrome). The concentrations of human and mouse sFasL proteins were measured using ELISA Kits (MBL or R\&D Systems, respectively), per the manufacturer's instructions. The lower detection limits were $75 \mathrm{pg} / \mathrm{ml}$ for human and $15 \mathrm{pg} / \mathrm{ml}$ for mouse sFasL.

\section{Human subjects and BAL protocol}

The human protocols were approved by the Human Subjects Review Committee of the University of Washington, and informed consent was obtained from the patients or their surrogates. The patient population, selection criteria, and BAL procedure and sample processing have been described (Table 2 and refs. 26, 58). The characteristics of the patients are summarized in Table 2. Normal volunteers were healthy, nonsmoking subjects (aged 18-50 years). Each aliquot of BAL fluid was thawed and used only once. When indicated, BAL fluid samples were concentrated by centrifugation at $5,000 \mathrm{~g}$ at $4^{\circ} \mathrm{C}$, using 5,000-Da molecular weight cut-off filter units (Millipore). When indicated, the BAL fluid samples were depleted of albumin and IgG with anti-human albumin and anti-human IgG antibodies coupled to agarose beads using the ProteoSeek Antibody-Based Albumin/IgG Removal Kit (Pierce). After 2 hours of incubation at room temperature, the beads were separated from the samples by centrifugation.

\section{Gel filtration of human BAL fluid}

BAL fluid samples were pooled and concentrated 6-fold, as described above. The BAL fluid proteins were separated according to molecular weight using high-pressure liquid chromatography with a SynChropak GPC 100 column $(250 \mathrm{~mm} \times 4.6 \mathrm{~mm}$; SynChrom $)$ at a flow rate of $1 \mathrm{ml} / \mathrm{min}$ in PBS. Each pooled sample was separated into 40 fractions. The column was calibrated using alcohol dehydrogenase $(150 \mathrm{kDa})$, bovine serum albumin (67 kDa), ovalbumin (43 kDa), chymotrypsinogen A (25 kDa), and ribonuclease $\mathrm{A}(13.7 \mathrm{kDa})$.

\section{Incubation of rh-sFasL in buman BAL fluid or with MPO- dependent oxidants}

Exogenous rh-sFasL (Peprotech Inc.) was incubated at $37^{\circ} \mathrm{C}$ in BAL fluid samples for 90 minutes, or in the MPO-dependent oxidation reaction mixture for 1 hour, or with $\mathrm{HOCl}$ for 30 minutes. The MPO-dependent oxidation reaction was carried out in phosphate buffer $(20 \mathrm{mM}$ sodium phosphate, $100 \mu \mathrm{M}$ diethylenetriaminepentaacetic acid [DTPA], $\mathrm{pH}$ 7.4), which was supplemented with $\mathrm{MPO}, \mathrm{H}_{2} \mathrm{O}_{2}$, and $\mathrm{NaCl}$ for the $\mathrm{MPO}-\mathrm{H}_{2} \mathrm{O}_{2}$-chloride system or $\mathrm{NaNO}_{2}$ for the MPO- $\mathrm{H}_{2} \mathrm{O}_{2}$-nitrite system, as indicated in each experiment. At the end of the incubation (in BAL fluid, MPO reaction fluid, or $\mathrm{HOCl})$, the oxidant scavenger L-methionine $(2.5 \mathrm{mM})$ was added to the samples to terminate oxidation. When indicated as a control, the reaction samples were incubated with $\mathrm{L}$-methionine prior to the addition of rh-sFasL to block oxidation of the protein. The concentrations of $\mathrm{H}_{2} \mathrm{O}_{2}$ and $\mathrm{HOCl}$ were determined spectrophotometrically $\left(\varepsilon_{240}=39.4 \mathrm{M}^{-1} \mathrm{~cm}^{-1}\right.$ and $\varepsilon_{292}=350 \mathrm{M}^{-1} \mathrm{~cm}^{-1}$, respectively). 


\section{Western blot of sFasL}

Samples were diluted in Laemmli sample buffer, with or without 2-ME $(5 \%)$, as indicated in each experiment. The proteins were separated by electrophoresis in 4-12\% SDS-PAGE gels (Lonza Biosciences Inc.), transferred to nitrocellulose membranes (Hybond-ECL NC, GE Healthcare), blocked with blocking buffer containing $0.2 \%$ SuperBlock (Pierce) and $0.05 \%$ Tween-20 at room temperature for 1 hour, and incubated overnight at $4{ }^{\circ} \mathrm{C}$ with primary detection antibody diluted in $0.2 \%$ SuperBlock $/ 0.05 \%$ Tween-20 buffer. After thoroughly washing with $1 \mathrm{x}$ PBS $0.05 \%$ Tween20 , the membranes were incubated for 1 hour at room temperature with HRP-conjugated secondary antibodies. Peroxidase activity was detected by chemiluminescence using Supersignal West Femto Maximum Sensitivity Substrate (Pierce).

\section{Incubation of rh-sFasL with urea}

The long form of rh-sFasL that was expressed in HEK cells was preoxidized by incubation with or without $\mathrm{HOCl}(50 \mu \mathrm{M})$ and then incubated in PBS, $\mathrm{pH} 7.4$, supplemented with or without urea $(8 \mathrm{M})$ at $37^{\circ} \mathrm{C}$ for 120 minutes. These rh-sFasL samples were diluted in Laemmli sample buffer containing 2-ME (5\%), separated by electrophoresis in 4-12\% SDS-PAGE gels, and analyzed by Western blotting.

\section{Incubation of rb-sFasL with DTT}

In order to determine whether disulfide bonds are involved in oxidation-mediated multimerization of human sFasL, the oxidized rh-sFasL (Peprotech Inc.) was incubated at $37^{\circ} \mathrm{C}$ in PBS containing DTT $(10 \mathrm{mM}$ or $20 \mathrm{mM}$ ). After 1 hour, iodoacetamide (IAM, $10 \mathrm{mM}$ ) was added to the reaction to prevent reformation of disulfide bonds and incubated at room temperature for another 30 minutes. As a control, rh-sFasL was diluted in PBS without either DTT or IAM. To stop the reaction and to reduce the content of DTT and IAM, the samples were diluted 1:5 in PBS and then spun in albumin-precoated centrifuge filter columns with a 3,000-Da molecular weight cut-off (Microcron Ultracel YM-3, Millipore) at 10,000 $\mathrm{g}$ for $25 \mathrm{~min}$ utes at $4^{\circ} \mathrm{C}$. The molecular weight of the sFasL subspecies was determined by SDS-PAGE, without reducing sample buffer, and Western blotting.

\section{Reduction of rh-sFasL in DMS}

In order to determine whether oxidation of methionine residues is involved in oxidation-mediated multimerization of human sFasL, we incubated oxidized rh-sFasL with DMS to reduce the methionine sulfoxide (oxidized methionine) back to methionine sulfide (nonoxidized methionine) (37). A rh-sFasL (Peprotech Inc.) was incubated with $0.1 \mathrm{M} \mathrm{DMS}$ and $10 \mathrm{M} \mathrm{HCl}$. The reaction was allowed to proceed at room temperature for 1 hour. To stop the reaction and reduce the content of DMS and $\mathrm{HCl}$, the samples were diluted 1:30 in 10X PBS, pH 7.4, spun in albumin-precoated centrifuge filter columns (3,000-Da molecular weight cut-off; Microcron Ultracel YM-3, Millipore) at $14,000 \mathrm{~g}$ for 25 minutes at $4^{\circ} \mathrm{C}$, and then diluted in $\mathrm{ddH}_{2} \mathrm{O}$. The molecular weight of sFasL subspecies was determined by SDS-PAGE, without reducing agents, and Western blotting.

\section{Incubation of rh-sFasL with MMP-7}

The long form of rh-sFasL was incubated at $37^{\circ} \mathrm{C}$ for 60 minutes with MMP-7 $(0.16,0.5$, or $1.5 \mu \mathrm{M})$ in buffer containing $20 \mathrm{mM}$ Tris, $\mathrm{pH} 7.6,5 \mathrm{mM}$ $\mathrm{CaCl}_{2}$, and $0.1 \mathrm{M} \mathrm{NaCl}$. After incubation, the samples were cooled to $4{ }^{\circ} \mathrm{C}$ and added to Jurkat cells or analyzed by Western blotting.

\section{LC-ESI-MS/MS}

Trypsin digestion. Samples containing exogenous human sFasL, previously incubated in ARDS BAL fluid, or the $\mathrm{MPO}-\mathrm{H}_{2} \mathrm{O}_{2}-\mathrm{Cl}^{-}$reaction mixture, or MMP-7 and endogenous sFasL affinity purified from ARDS BAL fluid were incubated and denatured in $4 \mathrm{mM}$ DTT, $50 \mathrm{mM}$ ammonium bicarbonate, $\mathrm{pH} 8.0$, and $5 \%$ acetonitrile for $15-20$ minutes at $70-80^{\circ} \mathrm{C}$. After cooling to room temperature, proteins were alkylated using IAM $(10 \mathrm{mM})$ for $15 \mathrm{~min}$ utes and then digested overnight at $37^{\circ} \mathrm{C}$ with sequencing grade-modified trypsin (Promega) (1:20 wt/wt, trypsin/protein). Digestion was halted by acidification with trifluoroacetic acid $(\mathrm{pH} 2-3)$. The tryptic peptides were desalted using a C18 column (Empore High Performance C18HD Extraction Disk Cartridges; $3 \mathrm{M}$ ), dried under vacuum, and then resuspended in $0.1 \%$ formic acid before analysis by mass spectrometry.

LC-ESI-MS/MS. LC-ESI-MS/MS analysis was performed in the positive ion mode with a Finnigan LCQ Deca XP ion trap mass spectrometer coupled to a quaternary Finnigan Surveyor HPLC (Thermo Electron Corp.). Tryptic peptides were separated using a Reversed-Phase Capillary HPLC Column (180 $\mu \mathrm{m} \times 10 \mathrm{~cm} ; 5-\mu \mathrm{m}$ particles; Biobasic 18, Thermo Electron Corp.), using solvent $\mathrm{A}(0.1 \% \mathrm{vol} / \mathrm{vol}$ formic acid in water) and solvent $\mathrm{B}(100 \%$ acetonitrile in $0.1 \%$ formic acid). The peptides were eluted using the following linear gradient: $0 \%-10 \%$ B over 10 minutes; $10 \%-35 \%$ B over 85 minutes; then $35 \%-80 \%$ B over 5 minutes at a flow rate of $2 \mu \mathrm{l} /$ minutes.

nanoLC-ESI-MS/MS. nanoLC-ESI-MS/MS analysis was performed in the positive ion mode with a Thermo-Finnigan LTQ linear ion trap mass spectrometer coupled to a Waters nanoAcquity UltraPerformance LC (UPLC) system. Peptides were separated on a nanoUPLC BEH130 C18 column $(100 \times 0.075 \mathrm{~mm}, 1.7 \mu \mathrm{m}$, Waters $)$, using solvent A $(0.1 \%$ formic acid in water) and solvent $\mathrm{B}\left(0.1 \%\right.$ formic acid in $\left.\mathrm{CH}_{3} \mathrm{CN}\right)$. Peptides were eluted using a linear gradient of $0 \%-35 \%$ solvent $\mathrm{B}$ over 60 minutes at a flow rate of $300 \mathrm{nl} / \mathrm{min}$.

\section{Cytotoxicity of sFasL in cultured cells}

Human Jurkat T Lymphocytes (TIB-152, clone E6-1, ATCC) and MLE-15 cells (mouse lung epithelial cell line provided by J. Whitsett, Cincinnati Children's Hospital, Cincinnati, Ohio, USA) were grown in RPMI-1640 medium supplemented with $10 \%$ or $4 \%$ heat-inactivated fetal bovine serum, respectively, at $37^{\circ} \mathrm{C}$ and $5 \% \mathrm{CO}_{2}$. Human SAECs (Lonza) were grown in small airway epithelial basal medium (SAGM), supplemented with SAGM SingleQuots (Lonza) at $37^{\circ} \mathrm{C}, 5 \% \mathrm{CO}_{2}$. For the bioassay experiments, Jurkat cells $\left(2 \times 10^{5}\right.$ cells/well), MLE- 15 cells (at $75 \%$ confluence), or SAECs (at $100 \%$ confluence) were seeded in Costar 96-well tissue culture plates (Corning Inc.) supplemented at a 50\% concentration with serum-free medium containing BAL fluid samples, rh-sFasL, or medium only. After incubation for 18 hours, the medium was removed, spun at $200 \mathrm{~g}$, and stored in individual aliquots at $-20^{\circ} \mathrm{C}$ for cytokine determinations. Cell viability was assessed using the alamarBlue assay (BioSource International), which incorporates a fluorometric indicator that detects mitochondrial activity. Data are shown as the percentage of cell death, which was calculated as follows: cell death $(\%)=100 \times([$ live cell fluorescence - experimental fluorescence] $/$ live cell fluorescence). Untreated live cell fluorescence corresponds to the fluorescence of cells in medium only. Finally, the cells were lysed, and caspase-3 activity was measured in each well using a Caspase-3 Fluorometric Assay Kit (Biovision). The fluorescence was measured using a CytoFluor II Fluorometer (PerSeptive Biosystems).

\section{Animals}

The animal protocols were approved by the Animal Care Committee of the VA Puget Sound Health Care System (Seattle, Washington, USA). Male C57BL/ 6 and B6.MRL-Fas $l p r / J$ mice (lpr mice) (The Jackson Laboratory), weighing $25-30 \mathrm{~g}$, were anesthetized with inhaled isoflurane, $2 \%-5 \%$, and treated once by intratracheal instillation of purified rh-sFasL $(25 \mathrm{ng} / \mathrm{g})$ or supernatants $(10 \mathrm{ng} / \mathrm{g})$ of HEK cells transfected with a cDNA encoding human sFasL. After the instillations, the mice were allowed to recover from anesthesia, returned to their cages, and provided with free access to food 
and water. The mice were euthanized at 16 hours after instillation with an intraperitoneal injection of pentobarbital $(120 \mathrm{mg} / \mathrm{kg})$ and exsanguinated by closed cardiac puncture. The thorax was opened rapidly, and the trachea was cannulated with a 20 -gauge catheter, the left hilum was clamped, and the left lung was removed and flash frozen in liquid nitrogen. The right lung was lavaged with 5 separate $0.5 \mathrm{ml}$ aliquots of $0.9 \% \mathrm{NaCl}$ containing $0.6 \mathrm{mM} \mathrm{EDTA}$ at $37^{\circ} \mathrm{C}$ and fixed by intratracheal instillation of $4 \%$ paraformaldehyde at a transpulmonary pressure of $15 \mathrm{~cm}$ of water and then embedded in paraffin. Frozen lung sections were prepared by inflating the right lung with intratracheal instillation of Tissue-Tek OCT (Sakura Finetek USA Inc.) (50\% vol/vol) in ribonuclease-free PBS. The lungs were then placed in cryomolds, covered with Tissue-Tek OCT, and frozen at $-80^{\circ} \mathrm{C}$. Cryosections were cut at $8 \mu \mathrm{M}$ thickness and mounted on glass slides.

\section{Analysis of mouse BAL fluid}

The BAL fluid samples were processed immediately for total and differential cell counts. Total white cell counts were performed with a hemacytometer, and differential counts were performed on cytospin preparations stained with the Diff-quick method (Andwin Scientific). A minimum of 200 cells were counted. The remainder of the lavage fluid was spun at $200 \mathrm{~g}$ for 30 minutes, and the supernatant was removed aseptically and stored in individual aliquots at $-80^{\circ} \mathrm{C}$. The total protein concentration in BAL fluid was measured by the bicinchoninic acid method (BCA assay; Pierce), and the concentration of IgM in BAL fluid was measured using ELISA (Bethyl Laboratories) per the manufacturer's instructions. The lower limit of detection of the IgM assay was $20 \mathrm{ng} / \mathrm{ml}$.

\section{Histological methods in mouse lung tissue}

Paraffin-embedded murine lung tissue sections (4- $\mu \mathrm{m}$ thick) were stained with hematoxylin and eosin for light microscopy. The TUNEL fluorescent staining for detection of DNA damage in situ was performed according to the manufacturer's instructions (Roche Diagnostics). Light, fluorescence, and differential interference contrast microscopy were performed using a Nikon Eclipse 80i microscope. Evaluation of lung tissue damage and measurement of TUNEL-positive cells were assessed in a blinded manner on 8 randomly generated visual fields at $\times 200$ magnification. Dual immunohistochemistry was used to compare TUNEL-positive and cytokeratin-positive cells (marker of epithelial cells) in the same frozen tissue sections. Briefly, the sections were fixed in acetone for 20 minutes at $4{ }^{\circ} \mathrm{C}$, and the TUNEL method was performed first. Then, the tissues were reblocked with Dako Serum-Free Protein Block (Dako) for 30 minutes at room temperature in the dark. The tissues were incubated with A555-fluorescent labeled mouse monoclonal pan-cytokeratin antibody (from Abcam, and labeled with Alexa fluor 555 dye) overnight at $4{ }^{\circ} \mathrm{C}$ in a moist chamber in the dark. After washing in PBS, the sections were covered with glass coverslips using glycerol and analyzed by light and fluorescence microscopy.

\section{Measurements in lung homogenates}

For cytokine and caspase- 3 activity measurements, the left lung was homogenized in lysis buffer containing $0.5 \%$ Triton X-100, $150 \mathrm{mM} \mathrm{NaCl}$,
$15 \mathrm{mM}$ Tris, $1 \mathrm{mM} \mathrm{CaCl}_{2}$, and $1 \mathrm{mM} \mathrm{MgCl}_{2}$ (pH 7.4) using a hand-held homogenizer. The homogenates were incubated for 30 minutes at $4{ }^{\circ} \mathrm{C}$ and then spun at $10,000 \mathrm{~g}$ for 20 minutes. For MPO measurements, the lungs were homogenized in $50 \mathrm{mM}$ potassium phosphate buffer ( $\mathrm{pH}$ 6.0) with $0.5 \%$ hexadecyltrimethyl ammonium bromide and $5 \mathrm{mM}$ EDTA and sonicated briefly at $4{ }^{\circ} \mathrm{C}$. MPO activity was measured in supernatants of lung homogenates using the Amplex Red fluorometric assay, per instructions from the manufacturer (Molecular Probes). Cytokines IL-1 $\beta$, IL-4, IL-6, IL-10, IL-12, KC, MIP-2, MCP-1, TNF- $\alpha$, and IFN- $\gamma$ were measured in lung homogenates using Fluorokine MultiAnalyte Profiling Kits (R\&D Systems) in a multiplex fluorescent bead assay (Luminex), per the manufacturer's instructions. The sensitivities of these immunoassays were $37.9 \mathrm{pg} / \mathrm{ml}$ for IL- $1 \beta, 11.25 \mathrm{pg} / \mathrm{ml}$ for IL-4, $10.53 \mathrm{pg} / \mathrm{ml}$ for IL-6, $7.47 \mathrm{pg} / \mathrm{ml}$ for IL-10, $23.83 \mathrm{pg} / \mathrm{ml}$ for IL-12, $13.08 \mathrm{pg} / \mathrm{ml}$ for KC, $6.69 \mathrm{pg} / \mathrm{ml}$ for MIP-2, $54.15 \mathrm{pg} / \mathrm{ml}$ for MCP-1, $6.82 \mathrm{pg} / \mathrm{ml}$ for TNF- $\alpha$, and $22.19 \mathrm{pg} / \mathrm{ml}$ for IFN- $\gamma$. Caspase-3 activity in lung homogenates was measured with the Caspase-3/CPP32 Fluorometric Assay Kit (Biovision), according to manufacturer's instructions. The fluorescence signal was measured using a fluorescent microplate reader.

\section{Statistics}

The results of the quantitative variables were expressed as mean \pm SD or plotted in scattered dot graphs showing the median. Differences among 3 or more groups were analyzed using 1-way ANOVA, followed by the Bonferroni's post-hoc tests for variables with normal distribution, or the KruskalWallis test, followed by the Dunn's test for those without a normal distribution. The means of 2 groups were compared using a 2-tailed unpaired Student's $t$ test. A logarithmic transformation $\left(\log _{10}\right)$ was used to reduce the heterogeneity of variances when these were significantly different. A $P$ value less than 0.05 was considered statistically significant. The statistical analyses were performed using GraphPad Prism 5.0 (GraphPad Software Inc.).

\section{Acknowledgments}

We thank John Ruzinski for his technical assistance with cytokine measurements and J. Whitsett for providing the MLE-15 cells. The mass spectrometry experiments were performed by $\mathrm{X}$. Fu and Y. Wang in the Mass Spectrometry Resource Core, Department of Medicine, University of Washington and the Puget Sound Blood Center. This work was supported in part by the Medical Research Service of the Department of Veterans Affairs and by grants HL-081764, HL-083044, HL-075381, and P30 DK-17047 from the NIH.

Received for publication September 11, 2010, and accepted in revised form December 8, 2010.

Address correspondence to: Thomas R. Martin, Pulmonary Research Labs, 151L, VA Puget Sound Medical Center, 1660 South Columbian Way, Seattle, Washington 98108, USA. Phone: 206.764.2219; Fax: 206.768.5289; E-mail: trmartin@u.washington.edu.
1. Rubenfeld GD, et al. Incidence and outcomes of acute lung injury. N Engl J Med. 2005; 353(16):1685-1693.

2. Bachofen M, Weibel ER. Structural alterations of lung parenchyma in the adult respiratory distress syndrome. Clin Chest Med. 1982;3(1):35-56.

3. Matthay MA, Robriquet L, Fang X. Alveolar epithelium: role in lung fluid balance and acute lung injury. Proc Am Thorac Soc. 2005;2(3):206-213.

4. Ashbaugh DG, Bigelow DB, Petty TL, Levine BE. Acute respiratory distress in adults. Lancet. 1967;2(7511):319-323.
5. Bardales RH, Xie SS, Schaefer RF, Hsu SM. Apoptosis is a major pathway responsible for the resolution of type II pneumocytes in acute lung injury. Am J Pathol. 1996;149(3):845-852.

6. Martin TR, Hagimoto N, Nakamura M, MatuteBello G. Apoptosis and epithelial injury in the lungs. Proc Am Thorac Soc. 2005;2(3):214-220.

7. Tang PS, Mura M, Seth R, Liu M. Acute lung injury and cell death: how many ways can cells die? AmJ Physiol Lung Cell Mol Physiol. 2008;294(4):L632-L641.

8. Matute-Bello G, et al. Essential role of MMP-12 in Fas-induced lung fibrosis. Am J Respir Cell Mol Biol.
2007;37(2):210-221.

9. Matute-Bello G, et al. Fas-mediated acute lung injury requires fas expression on nonmyeloid cells of the lung. J Immunol. 2005;175(6):4069-4075.

10. Matute-Bello G, et al. Recombinant human Fas ligand induces alveolar epithelial cell apoptosis and lung injury in rabbits. Am J Physiol Lung Cell Mol Physiol. 2001;281(2):L328-L335.

11. Matute-Bello G, Winn RK, Jonas M, Chi EY, Martin TR, Liles WC. Fas (CD95) induces alveolar epithelial cell apoptosis in vivo: implications for acute pulmonary inflammation. Am J Pathol. 
2001;158(1):153-161

12. Imamura R, et al. Fas ligand induces cell-autonomous NF-kappaB activation and interleukin-8 production by a mechanism distinct from that of tumor necrosis factor-alpha. J Biol Chem. 2004; 279(45):46415-46423.

13. Ponton A, Clement MV, Stamenkovic I. The CD95 (APO-1/Fas) receptor activates NF-kappaB independently of its cytotoxic function. J Biol Chem. 1996; 271(15):8991-8995.

14. Hagimoto $\mathrm{N}$, et al. Induction of interleukin-8 secretion and apoptosis in bronchiolar epithelial cells by Fas ligation. Am J Respir Cell Mol Biol. 1999;21(3):436-445.

15. Suda T, Takahashi T, Golstein P, Nagata S. Molecular cloning and expression of the Fas ligand, a novel member of the tumor necrosis factor family. Cell. 1993;75(6):1169-1178.

16. Nagata S, Golstein P. The Fas death factor. Science. 1995;267(5203):1449-1456.

17. Itoh $\mathrm{N}$, et al. The polypeptide encoded by the cDNA for human cell surface antigen Fas can mediate apoptosis. Cell. 1991;66(2):233-243.

18. Orlinick JR, Elkon KB, Chao MV. Separate domains of the human Fas ligand dictate self-association and receptor binding. J Biol Chem. 1997; 272(51):32221-32229.

19. Schulte M, et al. ADAM10 regulates FasL cell surface expression and modulates FasL-induced cytotoxicity and activation-induced cell death. Cell Death Differ. 2007;14(5):1040-1049.

20. Vargo-Gogola T, Crawford HC, Fingleton B, Matrisian LM. Identification of novel matrix metalloproteinase-7 (matrilysin) cleavage sites in murine and human Fas ligand. Arch Biochem Biophys. 2002; 408(2):155-161.

21. Kiener PA, et al. Human monocytic cells contain high levels of intracellular Fas ligand: rapid release following cellular activation. J Immunol. 1997;159(4):1594-1598.

22. Berg D, et al. Enforced covalent trimerization increases the activity of the TNF ligand family members TRAIL and CD95L. Cell Death Differ. 2007;14(12):2021-2034.

23. Schneider P, et al. Conversion of membranebound Fas(CD95) ligand to its soluble form is associated with downregulation of its proapoptotic activity and loss of liver toxicity. J Exp Med. 1998;187(8):1205-1213.

24. Holler N, et al. Two adjacent trimeric Fas ligands are required for Fas signaling and formation of a death-inducing signaling complex. Mol Cell Biol. 2003;23(4):1428-1440.

25. Shiraishi $\mathrm{T}$, et al. Increased cytotoxicity of soluble Fas ligand by fusing isoleucine zipper motif. Biochem Biophys Res Commun. 2004;322(1):197-202.

26. Matute-Bello G, et al. Soluble Fas ligand induces epithelial cell apoptosis in humans with acute lung injury (ARDS). J Immunol. 1999;163(4):2217-2225.

27. Matthay MA, Geiser T, Matalon S, Ischiropoulos H. Oxidant-mediated lung injury in the acute respiratory distress syndrome. Crit Care Med.
1999;27(9):2028-2030

28. Fink MP. Role of reactive oxygen and nitrogen species in acute respiratory distress syndrome. Curr Opin Crit Care. 2002;8(1):6-11.

29. Gole MD, et al. Plasma proteins modified by tyrosine nitration in acute respiratory distress syndrome. Am J Physiol Lung Cell Mol Physiol. 2000; 278(5):L961-L967.

30. Baldwin SR, Simon RH, Grum CM, Ketai LH, Boxer LA, Devall LJ. Oxidant activity in expired breath of patients with adult respiratory distress syndrome. Lancet. 1986;1(8471):11-14.

31. Sittipunt C, et al. Nitric oxide and nitrotyrosine in the lungs of patients with acute respiratory distress syndrome. Am J Respir Crit Care Med. 2001; 163(2):503-510.

32. Fu X, Kassim SY, Parks WC, Heinecke JW. Hypochlorous acid oxygenates the cysteine switch domain of pro-matrilysin (MMP-7). A mechanism for matrix metalloproteinase activation and atherosclerotic plaque rupture by myeloperoxidase. J Biol Chem. 2001;276(44):41279-41287.

33. Stadtman ER, Moskovitz J, Levine RL. Oxidation of methionine residues of proteins: biological consequences. Antioxid Redox Signal. 2003;5(5):577-582.

34. Zhu S, Ware LB, Geiser T, Matthay MA, Matalon S Increased levels of nitrate and surfactant protein a nitration in the pulmonary edema fluid of patients with acute lung injury. Am J Respir Crit Care Med.2001; 163(1):166-172.

35. Fu X, Kassim SY, Parks WC, Heinecke JW. Hypochlorous acid generated by myeloperoxidase modifies adjacent tryptophan and glycine residues in the catalytic domain of matrix metalloproteinase-7 (matrilysin): an oxidative mechanism for restraining proteolytic activity during inflammation. J Biol Chem. 2003;278(31):28403-28409.

36. Pitt AR, Spickett CM. Mass spectrometric analysis of $\mathrm{HOCl}-$ and free-radical-induced damage to lipids and proteins. Biochem Soc Trans. 2008; 36(pt 5):1077-1082

37. Shechter Y. Selective oxidation and reduction of methionine residues in peptides and proteins by oxygen exchange between sulfoxide and sulfide. J Biol Chem. 1986;261(1):66-70.

38. Albertine KH, et al. Fas and Fas ligand are up-regulated in pulmonary edema fluid and lung tissue of patients with acute lung injury and the acute respiratory distress syndrome. Am J Pathol. 2002; 161(5):1783-1796

39. Hashimoto S, Kobayashi A, Kooguchi K, Kitamura $\mathrm{Y}$, Onodera $\mathrm{H}$, Nakajima $\mathrm{H}$. Upregulation of two death pathways of perforin/granzyme and FasL/ Fas in septic acute respiratory distress syndrome. Am J Respir Crit Care Med. 2000;161(1):237-243.

40. Mizuta M, et al. Fas ligand released by activated monocytes causes apoptosis of lung epithelial cells in human acute lung injury model in vitro. Biol Pharm Bull. 2008;31(3):386-390.

41. Nakamura M, et al. Differential response of human lung epithelial cells to fas-induced apoptosis. Am J Pathol. 2004;164(6):1949-1958.
42. Kitamura Y, et al. Fas/FasL-dependent apoptosis of alveolar cells after lipopolysaccharide-induced lung injury in mice. Am J Respir Crit Care Med. 2001; 163(3 pt 1):762-769.

43. Tateda K, et al. Hyperoxia mediates acute lung injury and increased lethality in murine Legionella pneumonia: the role of apoptosis. J Immunol. 2003;170(8):4209-4216

44. Neff TA, et al. Relationship of acute lung inflammatory injury to Fas/FasL system. Am J Pathol. 2005; 166(3):685-694

45. Perl M, et al. Fas-induced pulmonary apoptosis and inflammation during indirect acute lung injury. Am J Respir Crit Care Med. 2007;176(6):591-601.

46. Fine A, Anderson NL, Rothstein TL, Williams MC, Gochuico BR. Fas expression in pulmonary alveolar type II cells. Am J Physiol. 1997;273(1 pt 1):L64-L71.

47. Fligiel SE, et al. Matrix metalloproteinases and matrix metalloproteinase inhibitors in acute lung injury. Hum Pathol. 2006;37(4):422-430.

48. Levine RL, Berlett BS, Moskovitz J, Mosoni L, Stadtman ER. Methionine residues may protect proteins from critical oxidative damage. Mech Ageing Dev. 1999;107(3):323-332.

49. Levine RL, Moskovitz J, Stadtman ER. Oxidation of methionine in proteins: roles in antioxidant defense and cellular regulation. IUBMB Life. 2000;50(4-5):301-307.

50 . Vogt W. Oxidation of methionyl residues in proteins: tools, targets, and reversal. Free Radic Biol Med. 1995; 18(1):93-105.

51. Stadtman ER, Van Remmen H, Richardson A, Wehr NB, Levine RL. Methionine oxidation and aging. Biochim Biophys Acta. 2005;1703(2):135-140.

52. Fransson JR. Oxidation of human insulin-like growth factor I in formulation studies. 3. Factorial experiments of the effects of ferric ions, EDTA, and visible light on methionine oxidation and covalent aggregation in aqueous solution. J Pharm Sci. 1997; 86(9): $1046-1050$

53. Siegel RM, et al. SPOTS: signaling protein oligomeric transduction structures are early mediators of death receptor-induced apoptosis at the plasma membrane. JCell Biol. 2004;167(4):735-744.

54. Henkler F, et al. The extracellular domains of FasL and Fas are sufficient for the formation of supramolecular FasL-Fas clusters of high stability. J Cell Biol. 2005;168(7):1087-1098.

55. Algeciras-Schimnich A, Shen L, Barnhart BC, Murmann AE, Burkhardt JK, Peter ME. Molecular ordering of the initial signaling events of CD95. Mol Cell Biol. 2002;22(1):207-220.

56. Cheng J, et al. Protection from Fas-mediated apoptosis by a soluble form of the Fas molecule. Science. 1994;263(5154):1759-1762.

57. Wortinger MA, et al. Fas ligand-induced murine pulmonary inflammation is reduced by a stable decoy receptor 3 analogue. Immunology. 2003; 110(2):225-233

58. Matute-Bello G, et al. Neutrophil apoptosis in the acute respiratory distress syndrome. Am J Respir Crit Care Med. 1997;156(6):1969-1977. 